\title{
1 Metabolite Damage and Damage-Control in a Minimal Genome
}

3 Drago Haas ${ }^{1 \&}$, Antje M. Thamm ${ }^{2 \%}$, Jiayi Sun ${ }^{2 \$}$, Lili Huang ${ }^{3 \#, ~ L i j i e ~ S u n ~}{ }^{4}$, Guillaume A.W.

4 Beaudoin $^{2 \dagger}$, Kim S. Wise ${ }^{4}$, Claudia Lerma-Ortiz ${ }^{2}$, Steven D. Bruner ${ }^{5}$, Marian Breuer ${ }^{6}$, Zaida

5 Luthey-Schulten ${ }^{7}$, Jiusheng Lin ${ }^{8}$, Mark A. Wilson ${ }^{8}$, Greg Brown ${ }^{9}$, Alexander F. Yakunin ${ }^{9}$, Inna

6 Kurilyak $^{10}$, Jacob Folz ${ }^{10}$, Oliver Fiehn ${ }^{10}$, John I. Glass ${ }^{4}$, Andrew D. Hanson ${ }^{2}$, Christopher S.

7 Henry $^{11,12 *}$ and Valérie de Crécy-Lagard ${ }^{1,13 *}$

$9{ }^{1}$ Department of Microbiology and Cell Science, University of Florida, Gainesville, FL 32611,

10 USA

$11{ }^{2}$ Horticultural Sciences Department, University of Florida, Gainesville, FL 3261, USA

$12{ }^{3}$ Food Science and Human Nutrition Department, University of Florida, Gainesville, FL 32611,

13 USA

$14{ }^{4}$ J. Craig Venter Institute, La Jolla, CA 92037, USA

$15{ }^{5}$ Chemistry Department, University of Florida, Gainesville, FL 32611, USA

$16{ }^{6}$ Maastricht Centre for Systems Biology (MaCSBio), Maastricht University, $6200 \mathrm{MD}$

17 Maastricht, The Netherlands

$18{ }^{7}$ Department of Chemistry, University of Illinois at Urbana-Champaign, Urbana, IL 61801, USA

$19{ }^{8}$ Department of Biochemistry and the Redox Biology Center, University of Nebraska, Lincoln,

20 NE 68588, USA

$21{ }^{9}$ Department of Chemical Engineering and Applied Chemistry, University of Toronto, Toronto,

22 ON M5S 3E5, Canada; Centre for Environmental Biotechnology, School of Natural Sciences,

23 Bangor University, Bangor, LL57 2UW, UK

$24{ }^{10}$ West Coast Metabolomics Center, UC Davis, Davis, CA 95616, USA

$25 \quad{ }^{11}$ Data Science and Learning, Argonne National Laboratory, Argonne, IL 60439, USA

$26{ }^{12}$ Consortium for Advanced Science and Engineering, The University of Chicago, Chicago, IL

27 60637, USA

$28{ }^{13}$ University of Florida Genetics Institute, Gainesville, FL 32611, USA11

$29 \&$ Current address - Sanofi, 13 Quai Jules Guesde Vitry-sur-Seine 94400, France

$30 \%$ Current address - Havas Life Bird and Schulte, Urachstrasse 19, 79102 Freiburg im Breisgau,

31 Germany 
$32 \$$ Current address - Captozyme, 1622 NW 55th Place, Gainesville, FL 32653, USA

$33{ }^{\#}$ Current address - Lingnan Medical Research Center, Guangzhou University of Chinese

34 Medicine, Guangzhou, Guangdong, China, 510006

$35 \uparrow$ Current address -Ginkgo Bioworks, 27 Drydock Ave 8th Floor, Boston, MA 02210

* Corresponding Authors: Valérie de Crécy-Lagard vcrecy@ufl.edu and Christopher S. Henry

40 chenry@anl.gov

42 Target Journal : MBIO AAM fellow submission route

43 https://journals.asm.org/journal/mbio/fellows

44 Possible reviewers Carole Linster Vadim Gladyshev

45 Zoran Nikoloski https://www.mpimp-golm.mpg.de/13193/Zoran_Nikoloski

46 Pedro Mendes http://www.comp-sys-bio.org/pedro/Mendes.html

47 Paco Baroma-Gomez https://langebio.cinvestav.mx/en/Dr-Francisco-Barona

\section{Abstract}

51 Analysis of the genes retained in the minimized Mycoplasma JCVI-Syn3A genome established

52 that systems that repair or preempt metabolite damage are essential to life. Several genes with

53 known metabolite damage repair or preemption functions were identified and experimentally

54 validated, including 5-formyltetrahydrofolate cyclo-ligase, CoA disulfide reductase, and certain

55 hydrolases. Furthermore, we discovered that an enigmatic YqeK hydrolase domain fused to

56 NadD has a novel proofreading function in NAD synthesis and could double as a MutT-like

57 sanitizing enzyme for the nucleotide pool. Finally, we combined metabolomics and

58 cheminformatics approaches to extend the core metabolic map of JCVI-Syn3A to include

59 promiscuous enzymatic reactions and spontaneous side reactions. This extension revealed that

60 several key metabolite damage-control systems remain to be identified in JCVI-Syn3A, such as

61 that for methylglyoxal. 


\section{Introduction}

A foundational goal of synthetic biology was to create a minimal living organism by a

64 bottom-up approach (1). This goal was reached in 2016 with the creation of JCVI-Syn3.0 (2).

65 This organism, based on the blueprint of the ruminant pathogen Mycoplasma mycoides capri

66 serovar LC GM12, a Gram-positive bacterium, was built by combining DNA synthesis,

67 recombination, and genome transplantation techniques, and was designed to include only genes

68 that are required for survival or to support a reasonable growth rate (428 protein-coding genes

69 and 34 genes for RNAs) (2). The initial strain JCVI-Syn3.0 was extremely fragile and another

70 derivative with an additional 18 genes, JCVI-Syn3A was found to be more stable and was the

71 basis for the recently published metabolic model (3). Surprisingly, at the time of publication in

$722016, \sim 30 \%$ of the genes in JCVI-Syn3.0 could not be assigned a specific function. The initial

73 annotation has since been improved by manual curation (4), through the creation of a detailed

74 metabolic model (3) and through further in silico analyses (5) but $~ 85$ proteins with unknown or

75 just broadly defined function remain (Supplemental data S1). These unknowns cannot all be

76 missing parts of synthesis/breakdown pathways as the metabolic reconstruction only identified a

77 few such gaps, namely four metabolic and eight transport reactions (3).

A crucial area of metabolism that is left out of classical metabolic models is metabolite damage and repair. Enzymes make mistakes and metabolites can undergo spontaneous chemical reactions (for classical examples see reference (6)). These types of uncontrolled metabolite damage are ever-present and, when the resulting side-products have toxic effects, their accumulation can impose a fitness cost $(6,7)$. In recent years, it has been shown that many enzymes of formerly unknown function repair or pre-empt metabolite damage (8), that human diseases are caused by mutations in metabolite repair enzymes (9-11), and that pathway engineering can fail unless the necessary repair enzymes are installed (12). The emerging recognition of the nature and extent of metabolite damage and repair raised the question of the

87 importance of metabolite repair for the survival and growth of a minimal genome like JCVI-

88 Syn3/3A. By combining expert manual curation, comparative genomics, metabolomics, metabolic modeling, chemoinformatics, and experimental validation, we identified a set of

90 chemical damage reactions likely to occur in JCVI-Syn3 and some of the damage repair and

91 preemption activities that are encoded (or predicted) by this minimal genome. 


\section{Results and Discussion}

\section{Identification and experimental validation of homologs of known metabolite repair} enzymes

To identify the metabolite repair enzymes in JCVI-Syn3A we first manually scanned its proteome for homologs of known metabolite repair enzymes $(6,12,13)$ (see Supplemental data

99 S1 and supplemental methods). Several were found, as follows.

100 1. 5-FCL. 5-Formyltetrahydrofolate (5-CHO-THF) is a by-product of serine

101 hydroxymethyltransferase (SHMT) (14)(Fig. 1A) that inhibits folate-dependent enzymes and

102 must therefore be removed from the folate pool (15). Of various enzymes known to recycle 5-

103 CHO-THF (16), the most widespread is 5-formyltetrahydrofolate cyclo-ligase (5-FCL) (encoded

104 by the fau/ygfA gene (16) in E. coli). The JCVI-syn3A genome encodes a 5-FCL homolog

105 (JCVISYN3A_0443; 24\% identity over 93\% coverage); this gene was confirmed to encode an

106 active 5-FCL by a complementation assay. Specifically, an E. coli K12 $\Delta y g f A$ strain does not

107 grow on M9 minimal medium with $0.2 \%$ glucose as carbon source and $20 \mathrm{mM}$ glycine as sole

108 nitrogen source (16)(Fig. 1B). Expression of JCVISYN3A_0443 from a pUC19 derivative

109 plasmid allowed complementation of the growth phenotype (Fig. 1B). Note that the essentiality

110 of JCVISYN3A_0443 might not be due to the repair function alone, but also to a role in 5-CHO-

111 THF-polyglutamate salvage as a unique source of 5,10-methenyltetrahydrofolate-polyglutamate

112 (3).

113 2. Cellular thiol reductases. Like all organisms grown in the presence of oxygen, JCVI-syn3A

114 will encounter oxidative stress that can damage macromolecules. Maintaining protein and small-

115 molecule thiol groups in their reduced state is critical for cellular redox homeostasis (17).

116 Thioredoxin/thioredoxin reductase is the dominant protein thiol oxidoreductase system in many

117 organisms, using reducing equivalents ultimately derived from NAPDH $(18,19)$. The JCVI-

118 Syn3A genome encodes homologs of the thioredoxin system proteins (TrxB/JCVISYN3A_0819

119 and TrxA/JCVISYN3A_0065) that are most likely involved in reducing disulfide bonds in

120 proteins and have already been partially characterized in other Mycoplasma species (Fig. 2A)(20,

121 21). Both genes are essential (Supplemental data S1), supporting a key role of the TrxA/TrxB

122 system in disulfide bond reduction. Note, however, that thioredoxin is also the hydrogen donor 
123 to ribonucleotide reductase, and thus JCVISYN3A_0819 and JCVISYN3A_0065 may be essential due to this possible connection to deoxyribonucleotide biosynthesis $(20,22)$.

JCVI3_0887 was found to be a homolog of CoA disulfide reductase (CoADR), which is

126 proposed to be the major mechanism to maintain redox balance in certain bacteria (23). Because

$127 \mathrm{CoA}$ is required for several reactions in the JCVI-syn3A metabolic model and is predicted to be

128 imported from the medium, CoADR could be a minimalist solution to detoxify $\mathrm{H}_{2} \mathrm{O}_{2}$. We

129 therefore tested the CoADR activity of the JCVISYN3A_0887 protein in vitro.

130 JCVISYN3A_0887 was found to be an active CoAD reductase that operates well at

131 physiologically relevant $\mathrm{pH}(\mathrm{pH} 7.5)$ (24) with reasonable $K_{\mathrm{M}}(0.17 \mathrm{mM})$ and $k_{\text {cat }}\left(2.8 \mathrm{~s}^{-1}\right)$ values

132 (Fig. 2B). It had no detectable activity against oxidized glutathione or pantethine (Fig. 2C).

133 While we cannot eliminate the possibility that reduced glutathione is imported from the medium

134 and oxidized glutathione is exported, this is a less parsimonious solution to the redox balance

135 problem than the CoA-based solution above.

\section{Functional analysis of orphan HAD family proteins identifies a nucleotide phosphatase} with possible dual roles

Our second strategy to identify metabolite repair enzymes was based on the fact that

141 metabolite repair (8)Five genes encoding stand-alone members of the HAD (haloacid

142 dehalogenase) hydrolase family (25) were identified in the JCVI-Syn3A genome (Supplemental

143 data S1) and are conserved in the recently analyzed close mollicute relative Mesoplasma florum

144 L1 (26) (Table 1). Such HAD hydrolases often participate in metabolite repair or homeostasis,

145 as many damaged and/or toxic intermediates are phosphorylated (e.g. phosphosugars), and the

146 first step in their recycling or removal requires a phosphatase $(8,27)$.

147 Comparative genomic analysis of the stand-alone HADs did not point to clear functional

148 hypotheses, except for JCVISYN3A_0728, whose location in a predicted operon with triose-

149 phosphate isomerase and phosphoglycerate mutase suggested a role in sugar phosphate

150 metabolism (Table 1). Possible functions for the HAD family proteins included: 1) metabolite

151 repair enzymes on substrates to be identified; 2) missing phosphatases involved in primary

152 metabolism identified by the metabolic model such as sedoheptulose 1,7-bisphosphate

153 phosphatase or phosphatidate phosphatase; 3) nucleotide phosphatases involved in dNTP pool 
154 maintenance. To discriminate among these hypotheses, we combined biochemical, genetic, and 155 metabolomics studies.

156 All four of the HAD proteins that we were able to express in E. coli (JCVISYN3A_0066, 157 JCVISYN3A_0077, JCVISYN3A_0728, JCVISYN3A_0907) were tested for activity against a 158 panel of 94 phosphatase substrates (Supplemental Table S1) (28). The four proteins had 159 detectable activity against the general phosphatase substrate $p$-nitrophenyl phosphate $(p N P P)$ and 160 different sets of physiological substrates (Fig. S1). The JCVISYN3A_0728 enzyme was active 161 against a wide range of nucleoside and sugar phosphates, the JCVISYN3A_0907 and 162 JCVISYN3A_0077 enzymes were active against narrower ranges of sugar phosphates, and the 163 JCVISYN3A_0066 enzyme was active against FMN and CoA. That sugar phosphates are among 164 the best substrates of the JCVISYN3A_0728 enzyme is consistent with the genomicallypredicted role in sugar phosphate metabolism, but no specific function or substrate could be assigned. Note, however, that the 94-substrate panel did not include damaged sugar phosphates.

We attempted to delete HAD-encoding genes in the JCVI-syn3A host as described in the Methods section. We expected this to be possible because transposon bombardment of the JCVIsyn3A genome indicated all five HADs were quasi-essential (i.e., required for fast growth but not essential for viability) [(3) and Supplemental data S1]. Deletion mutants were readily

172 JCVISYN3A_0907 (Supplemental data S2). Attempts to delete JCVISYN3A_0710 using two 173 different methods were unsuccessful (Supplemental data S2). It could be that the deletion of this gene resulted in an extremely slow-growing strain that could not be recovered using these approaches, or that JCVISVN3A_0710 is in fact essential and the transposon insertions in the gene were artifacts. The fact that the same gene is also essential in M. florum (Table 1) would suggest that the latter hypothesis is correct

We observed no major differences in growth rates between JCVI_Syn3A and any of the

180 JCVI-Syn3A control were grown in SP4-KO medium and harvested at the same point of log181 phase growth. Three biological replicates were prepared and three technical replicates from each 182 biological sample were distributed and further pelleted/rinsed/flash frozen/stored. Untargeted 183 metabolomic analysis of the mutant samples was carried out to screen for a broad range of 184 possible metabolic disruptions. The extraction, detection, and analyses of the metabolites are 
185 described in detail in the Supplemental methods and Supplemental Tables S2 and S3. A total of 1864152 features were detected in JCVI-Syn3A samples using hydrophilic interaction liquid 187 chromatography (HILIC) and mass spectrometry (Supplemental data S3). Metabolites were 188 annotated using accurate mass in addition to matching experimental MS/MS spectra to MS/MS 189 library spectra (MS/MS match) and/or matching experimental peaks to an in-house accurate 190 mass/retention time library (m/z-RT match). In total 522 metabolites were annotated as known 191 metabolites in cultures of JCVI-syn3A and mutants of JCVI-syn3A. Of these annotated 192 metabolites, 70 had both MS/MS and m/z-RT matches, 324 were annotated based on MS/MS 193 matches, 100 were annotated based on m/z-RT matches, and 28 annotations had MS/MS matches 194 linked to a small number of candidate compounds (e.g., "hexose-phosphate", which could be 195 multiple phosphorylated six-carbon sugars) (Supplemental data S3). Technical variance was assessed by measuring 43 internal standard compounds, which are non-endogenous chemicals 197 added to each sample and had an average coefficient of variance of $8.9 \%$ (Supplemental data 198 S3).

Partial least squares discriminant analysis was used to find the variable importance in 200 projection (VIP) scores of each annotated metabolite. The fifteen metabolites with the highest 201 VIP scores (Fig. 3 and Fig. S3) showed little contamination from media, as determined by 202 chemical analysis of unused media along with mutant samples. Most of these metabolites were 203 below the limit of detection in unused media, and most of the rest were present at much lower 204 abundance in media than in samples (>30-fold higher in samples compared to media) suggesting 205 little to no contamination from residual media in samples (Supplemental data S3). Two 206 metabolites (cytidine and thiamine) were found at similar abundance in media as compared to 207 samples, suggesting these two compounds may be influenced by media contamination.

Within this group of 15 metabolites with high VIP scores, the JCVISYN3A_0728

210 knockout showed significantly higher abundance of glycerophosphate, oleoyl lysophosphatidic 211 acid, and palmitoylglycerol as compared to other genotypes (Fig. 3 and Fig. S3). We were not 212 able to determine which form of glycerophosphate was increased, although the 3-phosphate is a 213 priori more likely, being found in the metabolic model as a cardiolipin metabolism intermediate 214 that is synthesized via phosphorylation of imported glycerol by GlpK (JCVISYN3A_0218). As 215 alpha-lysophosphatidic acid is produced from glycerol phosphate (3), these results suggest that 
216 the JCVISYN3A_0728 hydrolase could be involved in hydrolyzing glycerol phosphate (or a

217 derivative thereof).

The JCVISYN3A_0066 knockout had significantly higher deoxyuridine monophosphate,

220 inosine monophosphate, and deoxyinosine monophosphate, and lower thiamine and fructose-1-

221 phosphate abundance as compared to other mutants (Fig. 3 and Fig. S3). It is therefore possible

222 that JCVISYN3A_0066 is one of the phosphatases previously found to hydrolyze the

223 mononucleotides GMP, dAMP, dGMP, dUMP, and dTMP that were not identified in the

224 metabolic reconstruction (3)and further biochemical characterization is needed.

Comparative genomic approaches uncover a possible metabolite repair phosphatase

Comparative genomic analyses allowed us to propose a function for the YqeK HD family

228 phosphohydrolase that is fused to nicotinic acid mononucleotide adenylyltransferase (NadD) in

229 most mycoplasmas and strongly physically clustered with NadD in many other gram-positive

230 organisms (29) (Fig. 4 and S4A). This strong association led us to propose that the YqeK protein

231 repairs mistakes made by the NadD enzyme. The canonical activity of NadD is to adenylate

232 nicotinate-ribonucleotide (NaMN) using ATP as a donor of the AMP moiety (Fig. 4A).

233 However, if another NTP or the deoxy-form of ATP is used by mistake, this would create an

234 erroneous intermediate that would need to be hydrolyzed. We therefore expressed the

235 JCVISYN3A_0380 gene in E. coli as well as a variant encoding a His230Ala mutation (Fig.

236 S4B). (The mutation of this residue, predicted to be critical for the phosphatase activity, was

237 designed to stop product hydrolysis interfering with measurement of NadD activity.) The NadD

238 protein of Bacillus subtilis was also expressed and purified as a benchmark. The mutant

239 JCVISYN3A_0380 protein and B. subtilis NadD were then tested for in vitro activity with

240 various nucleoside triphosphates as substrates. As shown Fig. 5A, we found that the adenylation

241 activity of the JCVISYN3A_0380 His230Ala mutant was quite non-specific and actually worked

242 better with dATP, CTP, or UTP than with the natural substrate, ATP, whereas the B. subtilis

243 enzyme strongly preferred ATP. The JCVI-syn3 NadD enzyme can therefore readily form

244 deoxy-adenosine, -cytidine, or -uridine analogs of the NAD precursor nicotinate adenine

245 ainucleotide (NaAD) (which can presumably be converted to potentially inhibitory analogs of

246 NAD and NADP). 
We then tested the JCVI-Syn3 YqeK domain for phosphatase activity using the different $\mathrm{NaAD}$ analogs that could be produced by the JCVI-syn3A NadD enzyme. As shown Fig. 5B, the YqeK domain has activity towards the cytosine (NaCD) and uracil (NaUD) analogs of NaAD that is as high or higher than that against NaAD itself (which agrees with the preference of the NadD domain to make these analogs).

In order to assess the relative binding capacity for predicted substrates for YqeK, we docked the proposed molecules into the structure. The active site was defined at the di-iron site

254 and molecules were modeled with phosphate groups coordinated to the metals as seen in representative structures (PDB codes: CCG3, $2 \mathrm{O} 08$ and 2OGI). The conformations of the liganded substrates were optimized through rounds of energy minimization. The results show that the active site readily accommodates 2 '-deoxy-NaAD (Fig. S4C) and interactions of the adenosine and nicotinate moieties are consistent with other NaAD-binding proteins (e.g., PDB 1NUQ and 2QTR). Of note, a conserved Tyr82 clashes with the 2 '-hydroxyl of NaAD, not present in 2'-deoxy-NaAD.

In testing various possible substrates, we found the YqeK domain also had high activity

262 against 8-oxo-GTP, although judging from relative activities with $0.05 \mathrm{mM}$ and $0.5 \mathrm{mM}$ substrate, the $K_{\mathrm{M}}$ is likely higher than for the other substrates tested (Fig. 5B). Consistent with this finding, we showed that the genes encoding the JCVI-syn3A NadD-YqeK fusion can partially complement the $E$. coli mut $T$ high mutation rate phenotype (measured as Rif $^{\mathrm{R}}$ ratios) (Fig. 5C). The partial complementation was also observed when expressing the YqeK domain alone, but not the NadD domain alone. Finally, it was recently shown that YqeK of grampositive bacteria such as B. subtilis or M. pneumoniae are members of a novel diadenosine

270 YqeK is a versatile phosphatase with several functional roles. Unfortunately, the genetic

271 dissection we performed in JCVI-Syn3A did not allow us to confirm any of these roles in vivo.

272 Indeed, the available transposon insertion data ((3)and Supplemental data S1) suggested that the

273 NadD domain is essential and the YqeK domain is quasi-essential as a few hits in the YqeK

274 region of the gene were detected in the first Tn round, these disappeared after the fourth round of

275 growth. We were unable to isolate a JCVISYN3A_0380 gene deletion mutant in JCVI-Syn3B

276 despite several attempts. We were, however, able to construct a derivative that contains the

277 His230Ala point mutation inactivating the YqeK activity (Supplemental data S2) that did not 
show any growth rate defect or any obvious metabolite imbalance (Fig. S2). Further studies will accordingly be needed to define the role of the YqeK proteins in metabolism.

\section{Metabolomics-driven exploration of damage and repair chemistry in JCVI-Syn3}

Thus far, all of our damage and repair cases began with an analysis of specific classes of genes in the JCVI-Syn3A genome, and from these cases we see clear instances of metabolite damage and repair occurring in the JCVI-Syn3A strain. But do these examples represent isolated exceptions, or are they the tip of an iceberg of uncharacterized metabolic chemistry occurring even in the simplest organism that can currently be constructed? To gain insights into this question, we applied a more systematic exploratory approach that started with the metabolomics data generated from our JCVI-Syn3A cell samples (see Supplemental Table S3).

We focused this analysis specifically on the set of 480 metabolites observed in these samples that satisfied two criteria: (1) the mass-spectrum-observed metabolite was confidently identified with a fully defined molecular structure; and (2) the metabolite was at least as abundant in the JCVI-Syn3A cells as in the growth medium. . Supplemental Table S4E contains

293 this list of 480 metabolomics peaks filtered from the full set of metabolomics data provided in

294 Table S3. We next compared the 480 identified peaks to the 33,978 compounds in the

295 ModelSEED database (31), which includes all of KEGG (32) and MetaCyc (33), resulting in 217

296 (45\%) matches (see Supplemental Table S4E). We next compared the 480 identified peaks to the 33,978 compounds in the ModelSEED database (32), which includes all of KEGG (33) and

298 MetaCyc (34), resulting in 217 (45\%) matches (see Supplemental Table S4E). This analysis

299 revealed that over half the observed metabolites fall outside current biochemistry databases, and

300 that even for compounds that do occur in existing databases, they take part in pathways that are

301 not included in the current representation of JCVI-Syn3 metabolism. To predict potential

302 chemical routes to as many of the observed metabolites as possible without limiting our search to

303 known chemistry or straying too far from known JCVI-Syn3 metabolism, we applied the

304 cheminformatics tool PickAxe (34). This tool applies generalized reaction rules to predict

305 potential novel reactions that a given set of metabolites (in this case, all JCVI-Syn3 metabolites)

306 may undergo given known spontaneous (7) and enzymatic $(35,36)$ chemical mechanisms. We

307 started our PickAxe exploration with the 304 metabolites included in the JCVI-Syn3A model and 308 applied the PickAxe algorithm for multiple iterations to allow for the generation of multistep 
pathways (see methods). We used both spontaneous and enzymatic reaction rules in the PickAxe expansion, enabling prediction of pathways comprised of a mixture of spontaneous and enzymatic reaction steps (as is the case with damage and repair pathways). In the initial iterations of the PickAxe algorithm, we discovered an increasing number of compounds generated that matched our observed metabolites, but after six iterations, these hits tapered off to just one new compound produced that matched an observed metabolite (blue line in Fig. 6). Interestingly, the number of compounds predicted by PickAxe that matched known biochemistry in the ModelSEED database (green line in Fig. 6) followed a similar trend. We halted the PickAxe expansion at this stage given the diminishing returns in useful or recognizable chemistry being generated. Overall, the final chemical network generated by PickAxe included 33,934 compounds, 61,939 reactions, and matched a total of 182 distinct metabolites (including the original 57 matching the JCVI-Syn3 model) and 1090 ModelSEED compounds (see Supplemental data S4C-D).

The network generated by PickAxe represents a pool of hypothetical chemistry possible given our reaction rules and the compounds in the JCVI-Syn3A model. We then used a new flux

324 balance analysis formulation, called metabo-FBA, to select a minimal subset of these reactions

325 that can connect the functioning JCVI-Syn3A model to as many observed metabolites as possible

326 using mass and energy balanced pathways (see Methods). Because we are working with a

327 minimal genome with limited enzymatic diversity and the present study specifically focused on

328 metabolite damage, we favored solutions that involved as many reactions generated by

329 spontaneous reaction rules as possible. Using this approach, we were able to produce a predicted

330 flux profile that succeeded in simultaneously pushing flux through reactions involving

331 compounds that matched 182 distinct observed metabolites (see solution depicted in Fig. 7 and

332 data in Supplemental data S4A and E). This solution included 145 (58\%) of the 252 reactions in

333 the JCVI-Syn3 model (purple reactions in Fig. 7), 129 additional ModelSEED reactions

334 (primarily predicted enzymatic reactions; green reactions in Fig. 7), 84 novel enzymatic reactions

335 (blue reactions in Fig. 7), and 74 novel spontaneous reactions (red reactions in Fig. 7) (data in

336 Supplemental data S4A). The fixed image of our flux solution depicted in Fig. 7 is of limited

337 value for permitting a detailed exploration of the fluxes, so we are also including all data files

338 and instructions needed to replicate this view in a fully functioning dynamic Escher map (see 
Supplemental data S5). Also, the fully expanded version of the JCVI-Syn3A model used to generate this flux solution is provided in SBML and JSON format in Supplemental data S5. metabolomics data based on known and novel biochemical reactions. It is unlikely that this solution is completely correct, but the true solution must make use of similar chemistry, start

344 with the same initial high-confidence JCVI-Syn3A compounds, and produce the same observed metabolic intermediates, meaning the true solution cannot depart too significantly from our selected solution. Thus, while we cannot define exact mechanisms for producing observed metabolites from this analysis, we can observe significant chemical trends that reveal insights into areas of limited understanding of metabolic chemistry and the role of spontaneous reactions in that chemistry.

Looking at the map broadly (Fig. 7), it is immediately apparent that there are hotspots of intense chemical expansion (adenine, cytosine, sugars, pyruvate, amino acids, central carbon trunk reactions, CoA) and other regions with little or no expansion (deoxynucleotides, guanine,

354 thymidine, THF, riboflavin, NAD). This likely has to do with the concentration and reactivity of the associated compounds. For example, deoxynucleotides lack a chemically active hydroxyl group present in standard nucleotides. Many of the intensely branching compounds represent high concentration metabolic starting points (e.g., sugars), end points (amino acids), and high flux intermediates (e.g., pyruvate). Given their higher concentrations, it is more likely that metabolomics will detect these compounds and their derivatives, and that these compounds will undergo additional chemistry.

The large number of ModelSEED reactions and the many predicted novel enzymatic reactions proposed by this approach represent previously unannotated but potential promiscuous side activities of existing annotated gene products in JCVI-Syn3A. The extensive metabolomic evidence for the presence of the products of these reactions points strongly to the presence of the 365 reactions themselves. This is best exemplified by the cluster of ModelSEED reactions expanding 366 from the glucose-6-phosphate (g6p) node of the JCVI-Syn3A model (see Fig. 7A). These 367 reactions involve phosphorylation and hydrolysis interconverting many different sugars and 368 polysaccharides, all of which have evidence for existence in our metabolomics data. While the 
model only contains reactions for glucose as a representative sugar, it is likely that this model and many other similar models are substantially understating the promiscuity of these enzymes. method involve a mixture of database reactions, predicted spontaneous reactions, and novel enzymatic reactions (30/50 total pathways). Any analysis that focused on only one or even two of these three reaction sources would explain a far smaller number of observed metabolites due to holes and dead-ends in the predicted pathways. A complete understanding of metabolism requires all three reaction data sources.

Another significant trend is the large portion of new predicted chemistry surrounding amino acids. A significant number of observed metabolomics peaks relate to amino acid derivatives, including numerous dipeptides and acetylated amino acids (see Fig. 7B). The dipeptides primarily serve as nutrients for the JCVI-syn3 strain, which contains the peptidases needed to degrade these compounds (a large number of the ModelSEED reactions added by our metabo-FBA approach relate to dipeptide transport and degradation). However, the acetylated amino acids are interesting as only 7 out of 10 of these compounds were found in any biochemistry databases, which also lacked spontaneous reactions for producing these compounds. Yet, metabolomics evidence was found for all 10 compounds being present in the JCVI-Syn3A strain. The metabo-FBA approach added 10 predicted spontaneous acetylation reactions, using acetyl-phosphate as a donor, based on PickAxe predictions. This demonstrates how readily acetylation occurs in these systems, either by spontaneous action or by promiscuous enzyme activity, and it highlights the particular vulnerability of amino acids to this acetylation. In addition, these results further support certain hypotheses made previously about the main metabolic network of JCVI-Syn3A (3) both with regard to acetyl phosphate as well as the

392 enzymes producing/consuming it. The in vivo essentiality of phosphate acetyltransferase

393 (JCVISYN3_0229) and acetate kinase (JCVISYN3_0230) was puzzling, given that the preceding 394 genes in the pathway, the remaining subunits of pyruvate dehydrogenase (JCVISYN3_0227/8),

395 were found to be non-essential in vivo. It had been hypothesized that the two former enzymes 396 thus were not essential because acetate fermentation was essential for the cell, but rather because 397 buildups of acetyl-CoA or acetyl phosphate needed to be prevented, with acetyl phosphate a 398 known protein acetylation agent (37). 
Firstly, the current results hence support the role of acetyl phosphate as a biologically relevant acetylation agent in JCVI-Syn3A not only for free amino acids but also for proteins, as some of the identified amino acids had side chain acetylations. Secondly, the results also support the hypothesized essential role of acetate kinase as a means of preventing excess buildups of acetyl phosphate. Thirdly, if acetyl phosphate is indeed the acetylation agent at play, then this implies some source for acetyl phosphate/acetyl-CoA. Furthermore, if at least one of the two hypotheses for the essentiality of phosphate acetyltransferase and acetate kinase is correct (the latter now being supported by the current results), then this source would have to be intracellular production of acetyl-CoA rather than conversion of external acetate (as it would be these two enzymes which would then produce acetyl phosphate and acetyl-CoA in the first place). Production of acetyl-CoA in JCVI-Syn3A had not been certain previously, as the first subunit of pyruvate dehydrogenase and the related NADH oxidase had been removed in JCVI-Syn3A and the remaining components tentatively assumed to still be active. Alternatively, oxidation of

412 acetaldehyde to acetyl-CoA had been hypothesized as a possible function for the remaining 413 pyruvate dehydrogenase complex. The current results would suggest at least one of these two 414 hypothesized pathways to indeed produce acetyl-CoA - or there would have to be yet another 415 mechanism.

416 We are also particularly interested in using these analyses to understand the relative 417 prevalence, and thus importance, of our various proposed mechanisms for spontaneous 418 chemistry. One can examine prevalence in two ways, and our analyses explore both: (1) how 419 ubiquitous are the active sites that can undergo each given class of spontaneous reaction among 420 the metabolites present in JCVI-Syn3A; and (2) how often can each chemistry be observed to 421 happen based on metabolomics data. We can answer the first question by counting how many 422 reactions are generated by each spontaneous reaction operator in our PickAxe expansion of the 423 JCVI-Syn3A metabolites (orange bars in Fig. 8). From this, we find carbamylation to the most 424 dominant spontaneous mechanism with nearly 2000 reactions generated. Benzoquinone addition 425 reactions are also very prevalent with almost 1500 reactions generated. However, just because 426 chemistry occurs on a common active site does not mean it will be readily observed. Products 427 from the most visible chemistry are likely to accumulate and thus be observed in metabolomics 428 data. Thus, the reactions selected for addition by our metabo-FBA method represent the most 429 visible in terms of producing significant amounts of observable products. Counting the reactions 
selected by metabo-FBA for each of our spontaneous reaction mechanisms (gray bars in Fig.8) reveals acylation reactions as being the most common by far, with transamination also being

432 quite common. These results do not necessarily mean that all the other chemistry predicted by 433 our PickAxe analysis outside of those reactions involving or leading to observed metabolites is 434 not happening. Much of this chemistry may still be hidden from analysis if: (1) it only involves 435 compounds with concentrations below the threshold of detection; (2) it involves metabolites that 436 are not easily observed (e.g. highly unstable or volatile compounds); (3) it involves metabolites 437 that are not easily identified (e.g. new compounds with unknown fragmentation patterns and no 438 available standards). Thus, this difference between predicted chemistry and observed chemistry 439 could provide interesting targets for improving methods for observing and identifying 440 metabolites. Additionally, it is important to recognize that many PickAxe reaction types involve 441 co-substrates that are not present in the JCVI-Syn3A strain (e.g. benzoquinone or carbamoyl

442 transferase reactions), and thus it is expected that the products from these reaction types will not 443 appear in JCVI-Syn3A.

An example of an important intermediate metabolite that arises from and participates in spontaneous damage reactions but could not be observed using current metabolomics methods was methylglyoxal (see Fig. 7C). While methylglyoxal was not among the observed metabolites

447 due to small size and volatility, metabo-FBA added reactions involving this compound because it 448 leads to numerous downstream potential damage and repair reactions. A more detailed 449 discussion of methylglyoxal follows.

Methylglyoxal is necessarily generated from the triose phosphates formed by JCVI-

454 dependent GloA and GloB enzymes (39) is absent. Likewise, enzymes with minor

455 methylglyoxal-detoxifying activities, such as aldose reductases and keto-aldehyde reductases

456 (40-42) are not encoded in the JCVI-Syn3A genome. The only candidate enzyme that we

457 identified as potentially able to counter methylglyoxal-induced damage is JCVISYN3A_0400,

458 which encodes a homolog of DJ-1. The DJ-1 superfamily has several functionally distinct clades, 459 and phylogenetic analysis places JCVISYN3A_0400 in the YajL/DJ-1 clade (Fig. S6). The 
460 clades of the DJ-1 superfamily are not isofunctional and four subfamilies are found in E. coli 461 alone (encoded by the $h c h \mathrm{~A}, y a j \mathrm{~L}, y h b \mathrm{O}$ and $e l b \mathrm{~B}$ genes). uncertain, the functionally characterized DJ-1 superfamily proteins are involved in stress response and detoxification mechanisms (43). Some are thought to be deglycases (44), glyoxalases (45) and/or aldehyde-adduct hydrolases (46). We were not able to reproduce the previously reported glyoxal and/or methylglyoxal sensitivities of the $\Delta y a j \mathrm{~L} / \Delta h c h \mathrm{~A} E$. coli $\mathrm{K}-12$ BW25113 strain (44), but we did observe a defect both in its growth rate and yield (Fig. 9A and Fig. S7A). Expression of the E. coli yajL or JCVISYN3A_0400 genes in trans complemented this growth phenotype (Fig. 9A and Fig. S7A) suggesting JCVISYN3A_0400 was indeed in the same DJ-1 subgroup as YajL.

To test the hypothesis that JCVISYN3A_0400 is involved in methylglyoxal detoxification we expressed and purified the recombinant protein and measured its glyoxalase activity in vitro. As shown Fig. 9B the JCVISYN3A_0400 protein has very low but measurable methylglyoxalase activity $\left(k_{\mathrm{cat}}=0.025 \pm 0.002 \mathrm{sec}^{-1}, K_{\mathrm{M}}=1.23 \pm 0.3 \mathrm{mM}\right)$, far lower than obtained for the positive control proteins $S$. cerevisiae Hsp31 $\left(k_{\mathrm{cat}}=0.220 \pm 0.005 \mathrm{sec}^{-1}, K_{\mathrm{M}}=0.11 \pm 0.01 \mathrm{mM}\right)$ and somewhat lower than human DJ-1 $\left(k_{\mathrm{cat}}=0.073 \pm 0.002 \mathrm{sec}^{-1}, K_{\mathrm{M}}=0.34 \quad \pm 0.03 \mathrm{mM}\right)$. The $\sim 20$ $\mathrm{M}^{-1} \mathrm{sec}^{-1} k_{\mathrm{cat}} / K_{\mathrm{M}}$ value for JCVISYN3A_0400 is five to six orders of magnitude lower than that of glyoxalase I, the dedicated glutathione-dependent glyoxalase in most organisms (47). Even compared to other DJ-1 superfamily glyoxalases that have relatively low catalytic efficiency, JCVISYN3A_0400 is a poor enzyme. The lactate oxidase-coupled assay used here is specific to L-lactate, which should detect all the lactate produced by JCVISYN3A_0400, as previous reports indicate that DJ-1 clade enzymes produce only L-lactate (48). However, the more proficient Hsp31 glyoxalases produce racemic (D/L)-lactate (48), and thus the rate measured in this assay for $S$. cerevisiae Hsp31 is probably about half the true rate.

Because DJ-1 superfamily members have been reported to be generalist deglycases (49), we tested the deglycase activity of JCVISYN3A_0400 against the methylglyoxal-CoA hemithioacetal (Fig. S7B). CoA was used as the thiol because the absence of glutathione biosynthetic enzymes in JCVI-Syn3A means that CoA may be the principal small molecule thiol in the cell (see above). JCVISYN3A_0400 had no detectable deglycase activity against methylglyoxal-CoA hemithioacetal, while human DJ-1 had a low activity $\left(k_{\mathrm{cat}}=0.021 \pm 0.003 \mathrm{sec}^{-1}\right.$, 
$\left.K_{\mathrm{M}}=0.39 \pm 0.18 \mathrm{mM}\right)$. Therefore, JCVISYN3A_0400 appears unlikely to efficiently detoxify

492 methylglyoxal via either glyoxalase or deglycase activities if the in vitro rates are similar to the

493 in vivo activity of the protein. It is possible that JCVISYN3A_0400 and other DJ-1-type

494 glutathione-independent methylglyoxalases may have some unidentified positive effector in vivo

495 that could enhance their cellular activity, although there is currently no direct experimental

496 evidence for this. In summary, while results suggest that JCVISYN3A_0400 and YajL are iso-

497 functional, the molecular function of these proteins remain mysterious.

\section{Conclusion}

500 Metabolite damage arising from side-reactions of enzymes and spontaneous chemistry has often

501 been ignored or seen as a minor metabolic inconvenience that does not warrant investment in

502 enzymes to prevent or repair it (6). Biochemical, genetic, and engineering evidence

503 accumulating over the past decade has been changing this view $(6,7,10,12,50,51)$. The

504 biochemical and genetic results we present here constitute particularly persuasive additional

505 evidence by demonstrating that stripping a genome down to its barest essentials leaves

506 metabolite damage-control systems in place. Furthermore, our metabolomic and cheminformatic

507 results point to the existence of a network of metabolite damage and damage-control reactions

508 that extends far beyond the corners of it characterized so far. In sum, there can be little room left

509 to doubt that damage itself and the systems that counter it are mainstream metabolic processes.

\section{Methods}

\section{Bioinformatics}

513 The BLAST tools (52) and CDD resources at NCBI (http://www.ncbi.nlm.nih.gov/) (53) were

514 routinely used. Sequences were aligned using Clustal Omega (54) or Multialin (55).

515 Phylogenetic distribution was analyzed in the SEED database (56). Results are available in the

516 "YqeK" subsystem on the PubSEED server

517 (http://pubseed.theseed.org//SubsysEditor.cgi?page=ShowSpreadsheet\&subsystem=NadD-

518 YqeK_fusion_display). Physical clustering was analyzed with the SEED subsystem coloring tool

519 or the SeedViewer Compare Regions tool (56) and the clustering figure was generated with

520 GeneGraphics (57). Phylogenetic trees were constructed with Mega 6 (58). Student's t-test

521 calculations were performed using the VassarStats web-tools (http://vassarstats.net). 


\section{Prediction of novel potential chemistry using PickAxe}

526 Expanded chemistry was generated using the PickAxe app in KBase, as shown in this narrative:

527 https://narrative.kbase.us/narrative/29280. This app uses the open source RDKit package to

528 apply sets of SMARTS-based chemical reaction rules, derived from previously published

529 chemical damage (7) and enzyme promiscuity (34) studies, to an input set of compounds to

530 produce all possible reactions and products that might arise from that chemistry. This analysis

531 can be run iteratively through repeated application of the reaction rules to all new products that

532 arise from previous generations. We applied the PickAxe approach for six iterations, retaining all

533 compounds that matched the JCVI-Syn3A model, the ModelSEED database (31), or an observed

534 metabolite.

\section{Metabo-flux balance analysis to predict minimal reactions to reach observed metabolites}

537 In metabo-flux balance analysis (metabo-FBA), constraints are added to the standard FBA

538 formulation to force flux through one or more reactions involving an observed metabolite. In this

539 formulation, a variable is added for each observed peak $\left(\mathrm{p}_{\mathrm{i}}\right)$ and a variable is added for each

540 metabolite that has been mapped to the peak (because peaks lack stereochemistry, they may be

541 mapped to multiple possible stereoisomers). Next, a constraint is added stating that a peak cannot

542 be active unless one or more of its associated metabolites is active (where $\lambda_{i, j}$ is a mapping

543 variable equal to 1 if metabolite $j$ is mapped to peak $i$ and zero otherwise):

$$
p_{i} \leq \sum_{j}^{\text {Compounds }} \lambda_{i, j} m_{j}
$$

A constraint is also added stating that no metabolite can be active unless at least one

547 equal to 1 if metabolite $j$ is involved in reaction $k$ and zero otherwise):

$$
m_{j} \leq \sum_{k}^{\text {Reactions }} 100 \gamma_{j, k} v_{k}
$$

To maximize active metabolites, the objective of the problem is then set to maximize the sum of all $p_{i}$. While $p_{i}$ and $m_{j}$ can be specified as binary variables, it works equally well and is 
551 less computationally expensive to use continuous variables bounded between 0 and 0.1 . To avoid

552 the trivial solution of activating metabolites by pushing flux through both directions of reversible 553 reactions or around mass balanced flux loops, it is essential to also employ thermodynamics

554 constraints in some form in this formulation (59).

555

Synthesis of $\mathrm{NaAD}^{+}$analogs and nicotinic acid riboside (NaR)

$\mathrm{NaMN}(0.5 \mathrm{mM}), 4 \mathrm{mM} \mathrm{MgCl} 2,5$ units/ml yeast inorganic pyrophosphatase, $1 \mathrm{mg} / \mathrm{ml} \mathrm{BSA}$ and $2 \mathrm{mM}$ (d)NTP (dATP, CTP, GTP or UTP) were incubated with $150 \mu \mathrm{g}$ NadD-YqeKH230A enzyme overnight at $37^{\circ} \mathrm{C}$ in $20 \mathrm{mM}$ HEPES-KOH, pH 7.2, $100 \mathrm{mM} \mathrm{NaCl}, 0.2 \mathrm{mM}$ DTT, $1 \%$ glycerol. Assays were deproteinized using Amicon 10K cutoff centrifugal filters, concentrated in vacuo and purified by HPLC (Waters 2695 Separation module and Waters 2998 PDA detector) using a C18 column (Thermo Scientific Hypersil GOLD C18 $5 \mu \mathrm{m}, 250 \times 4.6 \mathrm{~mm}$ ) with a column guard with $20 \mathrm{mM}$ ammonium bicarbonate / acetic acid, $\mathrm{pH}$ 6.0. Purified $\mathrm{NaAD}^{+}$analogs were lyophilized and resuspended in $10 \mathrm{mM} \mathrm{HCl}, \mathrm{pH} 2.0$.

To synthesize a NaR standard, NaMN (10 mM) was dephosphorylated with 20 units CIP overnight at $37^{\circ} \mathrm{C}$. The mixture was deproteinized using Amicon $10 \mathrm{~K}$ cutoff centrifugal filters and used as a standard as is. The following extinction coefficients were used to quantify $\mathrm{NaAD}^{+}$ and its analogs: $\mathrm{NaAD}^{+} 19.4 \times 10^{-3} \mathrm{M}^{-1}$, dNaAD ${ }^{+} 19.4 \times 10^{-3} \mathrm{M}^{-1}, \mathrm{NaCD}^{+} 11.9 \times 10^{-3} \mathrm{M}^{-1}, \mathrm{NaGD}^{+}$ $16.4 \times 10^{-3} \mathrm{M}^{-1}, \mathrm{NaUD}^{+} 13.3 \times 10^{-3} \mathrm{M}^{-1}$. Extinction coefficients were based on published extinction coefficients of $\mathrm{NAD}^{+}$analogs (60) . To adjust for the nicotinic acid moiety, the difference of extinction coefficients of nicotinic acid $\left(4.2 \times 10^{-3} \mathrm{M}^{-1}\right)$ and nicotinamide $(2.78 \times$ $\left.10^{-3} \mathrm{M}^{-1}\right)(61)$ was added to those of the published NAD ${ }^{+}$analogs.

\section{Media, strains, and genetic manipulations}

575 All strains, plasmids and oligonucleotides used in this study are listed in Table S4 and Table S5.

576 Bacterial growth media were solidified with $15 \mathrm{~g} / \mathrm{l}$ agar (BD Diagnostics Systems) for the

577 preparation of plates. E. coli were routinely grown on LB medium (BD Diagnostics Systems) at

$578 \quad 37{ }^{\circ} \mathrm{C}$ unless otherwise stated. Transformations were performed following standard procedures

579 (62). IPTG (100 $\mu \mathrm{M})$, Ampicillin (Amp, $100 \mu \mathrm{g} / \mathrm{ml})$, Kanamycin (Km, $50 \mu \mathrm{g} / \mathrm{ml})$, 1-Arabinose 580 (Ara, 0.02-0.2\%), Chloramphenicol (Cm, $25 \mu \mathrm{g} / \mathrm{ml}$ ) and Rifampicin (Rif, $25 \mu \mathrm{g} / \mathrm{ml}$ ) were used 581 when appropriate. Bacterial M9 minimal medium (62), 0.4\% (w/v) glucose was used either with 
$582 \mathrm{NH}_{4} \mathrm{Cl}(20 \mathrm{mM})$ or glycine $(50 \mathrm{mM})$ as the nitrogen source. P1 transduction was performed

583 following the classical methods (63). The $\mathrm{Kan}^{\mathrm{R}}$ marker was eliminated from the BW2113

$584 \Delta y a j \mathrm{~L}:: \mathrm{Kan}^{\mathrm{R}}$ strain by the procedure described by Cherepanov and Wackernagel (64).

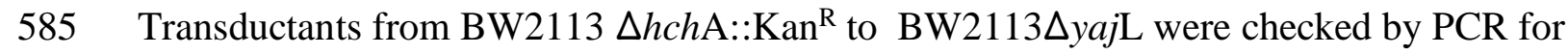

586 transduction of the $\Delta h c h \mathrm{~A}:: \operatorname{Kan}^{\mathrm{R}}$ allele into the recipient strains using primer pairs [DH492/493

587 (ext); DH494/495 (int) and DH480/481 (ext); DH482/483 (int)] respectively.

$588 \quad$ JCVI-syn3A is a near minimal bacterial cell first reported by Breuer et al. (3)that

589 contains a subset of the genes in Mycoplasma mycoides subspecies capri strain GM12.

590 Mycoplasmas were grown in SP4 broth (65) that contains 17\% KnockOut Serum Replacement ${ }^{\mathrm{TM}}$

591 instead of $17 \%$ fetal bovine serum and is referred to as SP4-KO as described in the supplemental

592 Methods.

\section{Construction and analysis of JCVI_Syn3A hydrolase gene deletion mutants.}

Construction of gene knockout mutants in JCVI-Syn3A was a multistep process, and two different protocols were used. Protocol I entailed CRISPR/Cas9 mediated removal of an individual target gene from a JCVI-Syn3A genome cloned as a yeast centromeric plasmid (YCp) in yeast strain VL648NCAS9_Syn3A that carries the cas9 gene in the yeast genome and expresses Cas9 constitutively $(2,66,67)$. Following the CRISPR/Cas9/homologous recombination with a donor DNA to re-circularize the JCVI-syn3A YCp, each mutated genome was transplanted by standard procedures $(68,69)$ in order to produce JCVI-syn3A bacteria lacking the respective target gene. Protocol I successfully removed gene JCVISYN3A_0728 but did not yield bacterial deletion mutants for any of the other four hydrolase genes. Protocol II, which is more complicated, was then used to make deletion mutants for the other hydrolase genes JCVISYN3A_0066, JCVISYN3A_0077, JCVISYN3A_0710, and. JCVISYN3A_0907. Using the yeast deletion constructs generated in JCVI-syn3A YCp using Protocol I, a second CRISPR/Cas9 was applied to install each respective hydrolase gene behind its native promoter between loxP sites in a non-essential region of the JCVI-syn3A genome. These four YCps were successfully transplanted to generate individual JCVI-syn3A bacteria, each with the respective target gene in essentially the same new location. We then transformed these bacterial relocation mutants, harboring the target gene between loxP sites, with a plasmid containing i) a Cre recombinase gene under transcriptional control of a mycoplasma promoter and (ii) a puromycin 
613 resistance gene. With this plasmid positioned between the same two loxP sites in the relocation

614 mutants, cells that were plated on Sp4 growth media containing puromycin, efficiently

615 exchanged the puromycin resistance cassette for genes JCVISYN3A_0066, JCVISYN3A_007,

616 and JCVISYN3A_0907, yielding the desired bacterial deletion mutants. We did not obtain a

617 JCVISYN3A_0710 deletion mutant. These protocols are described in detail in the

618 Supplemental data S2 file.

Inactivation of the hydrolase encoding domain of gene JCVISYN3A_0380 by converting

the His codon at position 230 to an Ala (His230Ala).

This was done using CRISPR/Cas9 as described previously to cut a yeast clone of the JCVI-syn3A YCp in gene JCVISYN3A_0379. Next, while the CRISPR cut JCVI-syn3A YCp

624 was still in yeast, a 1727 bp DNA molecule made using a multistage PCR that encoded gene 625 MMSYN1_0380 with the desired His to Ala mutation at codon 230 and flanked by sections of genes JCVISYN3A_0379 and JCVISYN3A_0381 was recombined into the linear JCVI-syn3A

627 YCp. The resulting mutated plasmid was installed in a Mycoplasma capricolum cell using 628 genome transplantation to create the JCVI-syn3A with the mutated JCVISYN3A_0380. The process is described in detail in the Supplemental data S2 file.

631 Plasmid constructions for expression JCVI-syn3A genes in E. coli

632 The sequences encoding all the JCVI-syn3A genes characterized in this study were codon-

633 optimized by the supplier (GenScript, Piscataway, NJ) for expression in E. coli. They were

634 synthesized with added restriction sites at the $5^{\prime}$ and $3^{\prime}$ ends and cloned in different vectors:

635 pUC19 for JCVISYN3A_0400, JCVISYN3A_0443 and JCVISYN3A_0887 at SphI and NcoI

636 sites; pUC57 for JCVISYN3A_0380 at NdeI and XhoI sites, or pET28a (JCVISYN3A_0728,

637 JCVISYN3A_0066, JCVISYN3A_0077, JCVISYN3A_907) at XbaI and XhoI sites which added

638 a C-terminal His-tag. The nucleotide sequences of all synthesized genes are given in

639 Supplemental Methods and the corresponding plasmids listed in Supplemental Table S4. The

640 sequences of the oligonucleotide primers used to subclone certain synthesized genes from

641 pUC19/pUC57 to other vectors are given Supplemental Table S5. JCVISYN3A_0887 was

642 cloned in the NcoI and BlpI sites of pET28 which added a C-terminal His tag after PCR 
643 amplification from pUC19-887 using the DH526 and DH527 primers. JCVISYN3A_0380 was

644 cloned in the NcoI and PstI sites of pBAD24 after PCR amplification from pUC57-380 using the

645 DH540 and DH541 primers. The same restriction sites were used to clone in pBAD24 the

646 fragment encoding only the $\mathrm{NadD}_{\mathrm{M}}$ domain (amplified by PCR from pUC57-380 using the

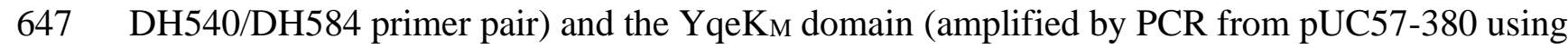

648 the DH585/DH541 primer pair). Design of the primers to separate the domains was based on

649 multiple alignment shown Fig. S4B. The JCVISYN3A_0380 was sub-cloned from pUC57-380

650 after digestion with NdeI and XhoI restriction sites and ligated into the matching restriction sites

651 of pET28a, which adds an N-terminal His tag.

$652 B s$ NadD Bs was PCR amplified with Phusion High-Fidelity DNA polymerase (New England

653 BioLabs) from Bacillus subtilis 168 genomic DNA using primers BsNadD_Fw and

654 BsNadD_Rv-XhoI digested with NdeI/XhoI and ligated into the matching sites of pET28b

655 (Novagen), which added a N-terminal His6-tag. The syn3.0 NadD-YqeK gene was synthesized

656 by Genscript. The YqeK domain was cloned into NdeI und XhoI restriction sites of pET28 after

657 PCR amplification using primers synYqeK-Fw_NdeI and synYqeK-Rv_XhoISTOP, which adds

658 a N-terminal His-Tag. The YqeK domain was cloned into NdeI und XhoI restriction sites of

659 pET28 after PCR amplification using primers synYqeK-Fw_NdeI and synYqeK-Rv_XhoISTOP,

660 which adds a N-terminal His-Tag. The JCVIJCVISYN3A_0380 H230A mutant was made by site

661 directed mutagenesis, using primers H230AFor/H230ARev to change the CAC codon (His) to

662 GCC (Ala), using Phusion polymerase. JCVISYN3A_0400 was cloned between the NdeI and

663 BamHI sites of bacterial expression vector pET15b after PCR amplification from pUC19-400

664 using primers DH611 and DH612. All constructs were verified by Sanger sequencing.

666 Mutation frequency assays for $E$. coli derivatives

667 Overnight cultures in LB with added antibiotics and arabinose (0.02\%) were diluted 100-fold in

668 the same conditions and grown for another $24 \mathrm{~h}$ before dilutions were plated on LB and LB

669 rifampicin $(25 \mu \mathrm{g} / \mathrm{ml})$ to calculate a mutation ratio (Number of colonies on Rif x dilution factor) /

670 (Number of colonies on LB x dilution factor).

671

672 Protein expression and purification and enzyme assays 
673 All characterized JCVI-syn3A encoded proteins were expressed as His-tagged variants in E. coli

674 and purified using $\mathrm{Ni}^{2+}$-NTA columns as described in Supplemental Methods. In vitro activity

675 assays for CoA disulfide reductase, for phosphatase with a range of substrates, NadD,

676 glyoxalase, and deglycase are described in detail in Supplemental Methods.

677 Acknowledgements. This work was funded by the National Science Foundation (Grants MCB

6781611846 to OF, MCB-1611952 to CH and MCB-1611711 to ADH and V dC-L, MCB 1840301, 679 MCB 1840320 and MCB 1818344 subcontracts to J.I.G.) and by the J. Craig Venter Institute. 
683 Table 1. Members of the HAD family of unknown function encoded by JCVI-Syn3

\begin{tabular}{|c|c|c|c|c|c|}
\hline Gene & Family & Essential & $\begin{array}{l}\text { Best } 3 \text { substrates } \\
\text { Activity in vitro* }\end{array}$ & Physical clustering & $\begin{array}{l}\text { M. florum ortholog } \\
\text { locus tag and } \\
\text { essentiality** }\end{array}$ \\
\hline JCVISYN3A_0066 & $\begin{array}{l}\text { Cof subfamily of IIB } \\
\text { subfamily of HAD } \\
\text { superfamily }\end{array}$ & no & $\begin{array}{l}\text { pNPP, FMN, } \\
\text { CoA }\end{array}$ & $\begin{array}{l}\text { Between 5S rRNA gene } \\
\text { and thioredoxin }\end{array}$ & Mfl169 (NE) \\
\hline JCVISYN3A_0077 & $\begin{array}{l}\text { Cof-like hydrolase, HAD } \\
\text { superfamily }\end{array}$ & no & Fru-1P, Ery-4P & Between $t s a D$ and $a s p S$ & Mfl614 (E) \\
\hline JCVISYN3A_0710 & $\begin{array}{l}\text { Cof subfamily of IIB } \\
\text { subfamily of HAD } \\
\text { superfamily }\end{array}$ & yes & Could not clone & $\begin{array}{l}\text { Between tRNA genes } \\
\text { and predicted } \\
\text { phosphonate transporter } \\
\text { genes }\end{array}$ & Mfl513 (E) \\
\hline JCVISYN3A_0728 & $\begin{array}{l}\text { HAD superfamily } \\
\text { hydrolase subfamily IIB, } \\
\text { protein }\end{array}$ & no & $\begin{array}{l}\text { GMP } \\
\text { XMP } \\
\text { 2-deoxy-glucose- } \\
6 \mathrm{P}\end{array}$ & $\begin{array}{l}\text { Between glycolysis } \\
\text { genes }\end{array}$ & Mfl503 (E) \\
\hline JCVISYN3A_0907 & $\begin{array}{l}\text { Cof-like hydrolase, HAD } \\
\text { superfamily }\end{array}$ & no & $\begin{array}{l}\text { N-acetyl-D- } \\
\text { glucosamine-6P } \\
\text { Fructose-1P } \\
\text { N-acetyl-D- } \\
\text { glucosamine-1P }\end{array}$ & $\begin{array}{l}\text { Between YidC and } \\
\text { choline kinase-like }\end{array}$ & Mfl680 (NE) \\
\hline
\end{tabular}

684 *Abbreviations in Table S1; ** (E), essential; (NE)=non-essential in M. florum 


\section{References}

1. Schwille,P. (2011) Bottom-up synthetic biology: engineering in a tinkerer's world. Science, 333, 1252-1254.

2. Hutchison,C.A., Chuang,R.-Y., Noskov,V.N., Assad-Garcia,N., Deerinck,T.J., Ellisman,M.H., Gill,J., Kannan,K., Karas,B.J., Ma,L., et al. (2016) Design and synthesis of a minimal bacterial genome. Science, 351, aad6253.

3. Breuer,M., Earnest,T.M., Merryman,C., Wise,K.S., Sun,L., Lynott,M.R., Hutchison,C.A., Smith,H.O., Lapek,J.D., Gonzalez,D.J., et al. (2019) Essential metabolism for a minimal cell. Elife, 8, e36842.

4. Danchin,A. and Fang,G. (2016) Unknown unknowns: essential genes in quest for function. Microb. Biotechnol., 9, 530-540.

5. Antczak,M., Michaelis,M. and Wass,M.N. (2019) Environmental conditions shape the nature of a minimal bacterial genome. Nat. Commun., 10, 3100.

6. Linster,C.L., Van Schaftingen,E. and Hanson,A.D. (2013) Metabolite damage and its repair or pre-emption. Nat. Chem. Biol., 9, 72-80.

7. Lerma-Ortiz,C., Jeffryes,J.G., Cooper,A.J.L., Niehaus,T.D., Thamm,A.M.K., Frelin,O., Aunins,T., Fiehn,O., de Crécy-Lagard,V., Henry,C.S., et al. (2016) 'Nothing of chemistry disappears in biology': the Top 30 damage-prone endogenous metabolites. Biochem. Soc. Trans., 44, 961-71.

8. de Crécy-Lagard,V., Haas,D. and Hanson,A.D. (2018) Newly-discovered enzymes that function in metabolite damage-control. Curr. Opin. Chem. Biol., 47, 101-108.

9. Becker-Kettern,J., Paczia,N., Conrotte,J.F., Zhu,C., Fiehn,O., Jung,P.P., Steinmetz,L.M. and Linster,C.L. (2018) NAD(P)HX repair deficiency causes central metabolic perturbations in

714 11. Veiga-da-Cunha,M., Van Schaftingen,E. and Bommer,G.T. (2020) Inborn errors of 715 metabolite repair. J. Inherit. Metab. Dis., 43, 14-24.

716 12. Sun,J., Jeffryes,J.G., Henry,C.S., Bruner,S.D. and Hanson,A.D. (2017) Metabolite damage 
and repair in metabolic engineering design. Metab. Eng., 44, 150-159.

13. Hanson,A.D., Henry,C.S., Fiehn,O. and de Crécy-Lagard,V. (2016) Metabolite Damage and Metabolite Damage Control in Plants. Annu. Rev. Plant Biol., 67, 131-52.

14. Stover,P. and Schirch,V. (1990) Serine hydroxymethyltransferase catalyzes the hydrolysis of 5,10-methenyltetrahydrofolate to 5-formyltetrahydrofolate. J. Biol. Chem. , 265, 1422714233.

15. Stover,P. and Schirch,V. (1993) The metabolic role of leucovorin. Trends Biochem. Sci., 18, $102-106$.

16. Jeanguenin,L., Lara-Nùñez,A., Pribat,A., Hamner Mageroy,M., Gregory 3rd,J.F., Rice,K.C., de Crécy-Lagard,V. and Hanson,A.D. (2010) Moonlighting glutamate formiminotransferases can functionally replace 5-formyltetrahydrofolate cycloligase. $J$. Biol. Chem., 285, 41557-66.

18. Berglund,O. and Holmgren,A. (1975) Thioredoxin reductase-mediated hydrogen transfer

17. Poole,L.B. (2015) The basics of thiols and cysteines in redox biology and chemistry. Free Radic. Biol. Med., 80, 148-57.

19. Thelander,L. (1967) Thioredoxin Reductase: Characterization of a homogeneous preparation froa Escherichia coli B. J. Biol. Chem., 242, 852-859.

20. Holmgren,A. (1985) Thioredoxin. Annu. Rev. Biochem., 54, 237-271.

21. Ben-Menachem,G., Himmelreich,R., Herrmann,R., Aharonowitz,Y. and Rottem,S. (1997) The thioredoxin reductase system of mycoplasmas. Microbiology, 143, 1933-1940.

22. Thelander,L. and Reichard,P. (1979) Reduction of Ribonucleotides. Annu. Rev. Biochem., 48,

741 23. DelCardayré,S.B., Stock,K.P., Newton,G.L., Fahey,R.C. and Davies,J.E. (1998) Coenzyme

742 A disulfide reductase, the primary low molecular weight disulfide reductase from

Staphylococcus aureus : purification and characterization of the native enzyme. J. Biol. Chem., 273, 5744-5751.

24. Benyoucef,M., Rigaud,J.-L. and Leblanc,G. (1981) The electrochemical proton gradient in Mycoplasma cells. Eur. J. Biochem., 113, 491-498.

25. Seifried,A., Schultz,J. and Gohla,A. (2013) Human HAD phosphatases: structure, 
mechanism, and roles in health and disease. FEBS J., 280, 549-571.

26. Lachance,J.-C., Matteau,D., Brodeur,J., Lloyd,C.J., Mih,N., King,Z.A., Knight,T.F., Feist,A.M., Monk,J.M., Palsson,B.O., et al. (2021) Genome-scale metabolic modeling reveals key features of a minimal gene set. Mol. Syst. Biol., 17, e10099-e10099.

27. Papenfort,K., Sun,Y., Miyakoshi,M., Vanderpool,C.K. and Vogel,J. (2013) Small RNAmediated activation of sugar phosphatase mRNA regulates glucose homeostasis. Cell, 153, 426-437.

28. Huang,L., Khusnutdinova,A., Nocek,B., Brown,G., Xu,X., Cui,H., Petit,P., Flick,R., Zallot,R., Balmant,K., et al. (2016) A family of metal-dependent phosphatases implicated in metabolite damage-control. Nat. Chem. Biol., 12.

29. Aravind,L. and Koonin,E. V. (1998) The HD domain defines a new superfamily of metaldependent phosphohydrolases. Trends Biochem. Sci., 23, 469-72.

30. Minazzato,G., Gasparrini,M., Amici,A., Cianci,M., Mazzola,F., Orsomando,G., Sorci,L. and Raffaelli,N. (2020) Functional characterization of COG1713 (YqeK) as a novel diadenosine tetraphosphate hydrolase family. J. Bacteriol., 202, e00053-20.

31. Seaver,S.M.D., Liu,F., Zhang,Q., Jeffryes,J., Faria,J.P., Edirisinghe,J.N., Mundy,M., Chia,N., Noor,E., Beber,M.E., et al. (2020) The ModelSEED Biochemistry Database for the integration of metabolic annotations and the reconstruction, comparison and analysis of

33. Krieger,C.J., Zhang,P., Mueller,L.A., Wang,A., Paley,S., Arnaud,M., Pick,J., Rhee,S.Y. and Karp,P.D. (2004) MetaCyc: a multiorganism database of metabolic pathways and enzymes. Nucleic Acids Res., 32, D438-42.

34. Jeffryes,J.G., Colastani,R.L., Elbadawi-Sidhu,M., Kind,T., Niehaus,T.D., Broadbelt,L.J.,

32. Kanehisa,M. and Goto,S. (2000) KEGG: kyoto encyclopedia of genes and genomes. Nucleic Acids Res., 28, 27-30.

35. Hatzimanikatis,V., Li,C., Ionita,J.A., Henry,C.S., Jankowski,M.D. and Broadbelt,L.J. (2005) Hanson,A.D., Fiehn,O., Tyo,K.E.J. and Henry,C.S. (2015) MINEs: Open access databases Exploring the diversity of complex metabolic networks. Bioinformatics, 21, 1603-9.

36. Henry,C.S., Broadbelt,L.J. and Hatzimanikatis,V. (2010) Discovery and analysis of novel 
metabolic pathways for the biosynthesis of industrial chemicals: 3-hydroxypropanoate. Biotechnol. Bioeng., 106, 462-73.

37. Weinert,B.T., Iesmantavicius,V., Wagner,S.A., Schölz,C., Gummesson,B., Beli,P., Nyström,T. and Choudhary,C. (2013) Acetyl-phosphate is a critical determinant of lysine acetylation in E. coli. Mol. Cell, 51, 265-272.

38. Phillips,S.A. and Thornalley,P.J. (1993) The formation of methylglyoxal from triose phosphates. Eur. J. Biochem., 212, 101-105.

39. Sukdeo,N. and Honek,J.F. (2008) Microbial glyoxalase enzymes: Metalloenzymes controlling cellular levels of methylglyoxal. Drug Metabol. Drug Interact., 23, 29-50.

40. Inoue,Y. and Kimura,A. (1995) Methylglyoxal and regulation of its metabolism in microorganisms. Adv. Microb. Physiol., 37, 177-227.

41. Misra,K., Banerjee,A.B., Ray,S. and Ray,M. (1996) Reduction of methylglyoxal in Escherichia coli K12 by an aldehyde reductase and alcohol dehydrogenase. Mol. Cell. Biochem., 156, 117-24.

42. Jagt,D.L.V., Robinson,B., Taylor,K.K. and Hunsaker,L.A. (1992) Reduction of trioses by NADPH-dependent aldo-keto reductases. Aldose reductase, methylglyoxal, and diabetic complications. J. Biol. Chem., 267, 4364-9.

43. Smith,N. and Wilson,M.A. (2017) Structural biology of the DJ-1 superfamily. Adv. Exp. Med. Biol., 1037, 5-24.

44. Richarme,G., Liu,C., Mihoub,M., Abdallah,J., Leger,T., Joly,N., Liebart,J.-C., Jurkunas,U. V, Nadal,M., Bouloc,P., et al. (2017) Guanine glycation repair by DJ-1/Park7 and its bacterial homologs. Science, 357, 208-211.

46. Matsuda,N., Kimura,M., Queliconi,B.B., Kojima,W., Mishima,M., Takagi,K., Koyano,F.,

47. Clugston,S.L., Barnard,J.F.J., Kinach,R., Miedema,D., Ruman,R., Daub,E. and Honek,J.F. (1998) Overproduction and characterization of a dimeric non-zinc glyoxalase I from 63. 
48. Choi,D., Kim,J., Ha,S., Kwon,K., Kim,E.H., Lee,H.Y., Ryu,K.S. and Park,C. (2014)

Stereospecific mechanism of DJ-1 glyoxalases inferred from their hemithioacetalcontaining crystal structures. FEBS J., 281, 5447-62.

49. Richarme,G., Abdallah,J., Mathas,N., Gautier,V. and Dairou,J. (2018) Further characterization of the Maillard deglycase DJ-1 and its prokaryotic homologs, deglycase 1/Hsp31, deglycase 2/YhbO, and deglycase 3/YajL. Biochem. Biophys. Res. Commun., 503, 703-709.

50. Van Schaftingen,E., Veiga-da-Cunha,M. and Linster,C.L. (2015) Enzyme complexity in intermediary metabolism. J. Inherit. Metab. Dis., 38, 721-727.

51. Golubev,A., Hanson,A.D. and Gladyshev,V.N. (2017) Non-enzymatic molecular damage as a prototypic driver of aging. J. Biol. Chem. , 292, 6029-6038.

53. Database resources of the National Center for Biotechnology Information (2018) Nucleic Acids Res., 46, D8-D13.

54. Li,W., Cowley,A., Uludag,M., Gur,T., McWilliam,H., Squizzato,S., Park,Y.M., Buso,N. and

52. Altschul,S.F., Madden,T.L., Schaffer,A.A., Zhang,J., Zhang,Z., Miller,W. and Lipman,D.J. Lopez,R. (2015) The EMBL-EBI bioinformatics web and programmatic tools framework. Nucleic Acids Res., 43, W580-4.

57. Harrison,K.J., de Crécy-Lagard,V. and Zallot,R. (2018) Gene Graphics: A genomic neighborhood data visualization web application. Bioinformatics, 34, 1406-1408.

58. Tamura,K., Stecher,G., Peterson,D., Filipski,A. and Kumar,S. (2013) MEGA6: Molecular Evolutionary Genetics Analysis version 6.0. Mol. Biol. Evol., 30, 2725-2729.

59. Henry,C.S., Broadbelt,L.J. and Hatzimanikatis,V. (2007) Thermodynamics-based metabolic flux analysis. Biophys. J., 92, 1792-1805.

60. Honjo,M., Furukawa,Y., Moriyama,H. and Tanaka,K. (1963) Synthesis of nicotinamide 
adenine dinucleotide analogs and their coenzymatic activities. Chem. Pharm. Bull. (Tokyo), 11, 712-720.

61. McLaren,J., Ngo,D.T.C. and Olivera,B.M. (1973) Pyridine Nucleotide Metabolism in Escherichia coli: III. Biosynthesis from alternative precursors in vivo. J. Biol. Chem., 248, 5144-5149.

62. Sambrook,J.E., Fritsch,E.F., Maniatis,T., Fritsch,E.F., Sambrook,J.E., Fritsch,E.F.,

63. Miller,J.H. (1972) Experiments in Molecular Genetics Cold Spring Harbor Laboratory Press,

64. Cherepanov,P.P. and Wackernagel,W. (1995) Gene disruption in Escherichia coli: TcR and KmR cassettes with the option of Flp-catalyzed excision of the antibiotic-resistance determinant. Gene, 158, 9-14.

65. Tully,J.G., Rose,D.L., Whitcomb,R.F. and Wenzel,R.P. (1979) Enhanced isolation of Mycoplasma pneumoniae from throat washings with a newly modified culture medium. $J$. Infect. Dis., 139, 478-482.

66. Kannan,K., Tsvetanova,B., Chuang,R.-Y., Noskov,V.N., Assad-Garcia,N., Ma,L., Hutchison Iii,C.A., Smith,H.O., Glass,J.I., Merryman,C., et al. (2016) One step engineering of the small-subunit ribosomal RNA using CRISPR/Cas9. Sci. Rep., 6, 30714.

67. Tsarmpopoulos,I., Gourgues,G., Blanchard,A., Vashee,S., Jores,J., Lartigue,C. and SirandPugnet,P. (2016) In-yeast engineering of a bacterial genome using CRISPR/Cas9. ACS Synth. Biol., 5, 104-109.

68. Lartigue,C., Glass,J.I., Alperovich,N., Pieper,R., Parmar,P.P., Hutchison,C.A., Smith,H.O. and Venter,J.C. (2007) Genome transplantation in bacteria: changing one species to another. Science, 317, 632-638.

69. Lartigue,C., Vashee,S., Algire,M.A., Chuang,R.-Y., Benders,G.A., Ma,L., Noskov,V.N., 


\section{$871 \quad$ Figure legends}

872 Figure 1. 5-FCL activity is encoded by JCVI_0400. (A) Enzymatic source and repair of 5-

873 CHO-THF. (B) Growth phenotype of a WT E. coli BW25113, $\Delta y g f$ A mutant and, $\Delta y g f$ A

874 mutant expressing JCVI_0443 gene on M9 minimal medium (0.4\% glucose) with (1) $20 \mathrm{mM}$

$875 \mathrm{NH}_{4} \mathrm{Cl}$ or (2) $50 \mathrm{mM}$ glycine as sole nitrogen source. Plates were incubated for 3 days at $37^{\circ} \mathrm{C}$.

Figure 2. Predicted and validated redox buffering systems in JCVI-Syn3. (A) Candidates for $\mathrm{H}_{2} \mathrm{O}_{2}$ detoxification systems of JCVI3, experimentally validated are in solid arrows, only the number of the locus tags are given, $\mathrm{P}$ is for protein, $\mathrm{R}$ is for small molecule. (B) CoADR Michaelis-Menten saturation curve for the determination of the Km and kcat for CoAD consumption. (C) CoADR is specific towards oxidized CoA with no activity towards other tested disulfides

Figure 3. Heatmap including 15 metabolites from JCVI-syn3A mutant metabolomic analysis with highest VIP scores. Samples and genotypes are represented in columns. High intensity measurements as compared to average intensity are red/yellow, and low intensity measurements are represented by green/blue

Figure 4. Predicted Hydrolase of unknown function is clustered or fused to NadD in many Firmicutes (A) Predicted NADP ${ }^{+}$synthesis pathway in JCVI-Syn3. (B) Physical clustering and fusions of $n a d D$ and $y k e K$ homologs in several gram-positive Bacteria. The RefSeq identifiers

892 for the yqeK genes used in descending order are: NP_975428.1, NP_390441.1, NP_372117.1,

893 NP_816490.1, YP_140036.1. (C) Docked model of 2-deoxy-NaAD bound to the $C$.

894 acetobutylicum YqeK (pdb code: 3CCG). The protein is shown in ribbon format (grey) with side

895 chains as lines, two iron atoms are shown as spheres bound to the diphosphate of

$896 \mathrm{dNaAD}$. Tyrosine 82 (green) is modeled as two conformations in the crystal structure and forms

897 a close interaction with the 2'carbon of dNaAD.

899 Figure 5. Biochemical analysis of the NadD and YqeK activities (A) Relative reaction rates of

900 Bacillus subtilis and JCVI syn3.0 NadD enzymes with NaMN and various nucleotides,

901 calculated as percentage of the canonical reaction with ATP for each NadD enzyme. Enzymes 
were incubated with $2 \mathrm{mM}$ NTP, $0.5 \mathrm{mM} \mathrm{NaMN}, 4 \mathrm{mM} \mathrm{MgCl} 2$ and $5 \mathrm{u} / \mathrm{ml}$ yeast inorganic pyrophosphatase for $5 \mathrm{~min}$ at $37^{\circ} \mathrm{C}$. $\mathrm{H} 230 \mathrm{~A}$ has the conserved $\mathrm{H}$ in the active site of the YqeK

904 domain mutated to ablate the HD activity and cleavage of nucleotides. (B) Activity of the

905 expressed JCVI syn3.0 YqeK domain with different substrates. YqeK (0.2 $\mu \mathrm{g})$ was incubated

906 with 0.5 or $0.05 \mathrm{mM}$ substrates, $1 \mathrm{mg} / \mathrm{ml} \mathrm{BSA}$ and $2.0 \mathrm{mM} \mathrm{MgCl}$ for $20 \mathrm{~min}$ at at $37^{\circ} \mathrm{C}$. Black

907 bars are data for $0.5 \mathrm{mM}$ substrates, white bars are data for $0.05 \mathrm{mM}$ substrates. (C) Mutation

908 ratio on LB rifampicin for strain $\Delta m u t \mathrm{~T}$ with empty vector (pBAD24), $\Delta m u t \mathrm{~T}$ with E. coli $m u t \mathrm{~T}$

909 in trans, $\Delta m u t \mathrm{~T}$ with either the nadD-yqeK fusion gene JCVI_0380, or the nadD or yqeK

910 domains alone. $* * *$ indicates a $\mathrm{P}$-value $<0.001$ with experiments performed with four biological

911 replicates and four technical replicates.

913 Figure 6. Generation of compounds matching observed peaks (blue line) or ModelSEED

914 compounds (green line) with each iteration of the PickAxe algorithm. Note, multiple compounds 915 can match the same peak if they are stereo isomers.

918 many observed peaks as possible. Grey reactions are inactive model reactions; purple reactions

919 are active model reactions; green indicates active ModelSEED reactions; red indicates active

920 predicted damage reactions; and blue indicates active predicted enzymatic reactions. All active

921 predicted spontaneous reactions and nearly all active model reactions are shown on the map;

922 some ModelSEED and predicted enzymatic reactions are excluded. The insets highlight

923 examples of promiscuous carbohydrate chemistry (A), prevalence of amino acid acetylation (B),

924 and spontaneous reactions mediated by the damage- causing metabolite methylglyoxal.

927 spontaneous reaction rules. The orange bars in the chart show the relative number of reactions

928 generated by each spontaneous reaction rule in the full PickAxe expansion of the JCVI-syn3

929 model. The gray bars show the relative number reactions generated by the same reaction rules

930 that were actually used to generate observed metabolites in the JCVI-syn3 strain by the metabo-

931 FBA analysis. To permit side-by-side comparison, the reaction counts are normalized by the

932 number of reactions associated with the most prevalent reaction rule in each set. The differences 
933 in the distributions highlight how the most promiscuous reaction rules (highest orange bars) are

934 not necessarily the most impactful on cell chemistry (highest gray bars).

935

936 Figure 9. Characterization of JCVI_0400. (A) Growth of WT, $\Delta y a j \mathrm{~L}, \Delta y a j \mathrm{~L} \Delta h c h \mathrm{~A}, \Delta y a j \mathrm{~L}$

$937 \Delta h c h \mathrm{~A}$ with $h c h A$ in trans and $\Delta y a j \mathrm{~L}$ LhchA with JCVI_0400 in trans. pUC19 was used as

938 empty vector. Each strain was tested in 5 replicates Plates were incubated 2 days at $37^{\circ} \mathrm{C}$ in $\mathrm{LB}$

939 with agitation in a Bioscreen C device. (B) Methylglyoxalase activity of JCV_0400 (MP DJ-1)

940 compared to human DJ-1 (HsDj1) and yeast Hsp31. Conversion of methylglyoxal to L-lactate

941 was measured in a coupled assay with L-lactate oxidase and Amplex red. JCVIsyn3_0400 is a

942 weak methylglyoxalase. Hsp31 produces racemic lactate, so the measured kobs is $\sim 1 / 2$ the true

943 rate and is higher than either close DJ-1 homolog. 


\section{Figure 1}

A 5,10-methenyl-THF

B

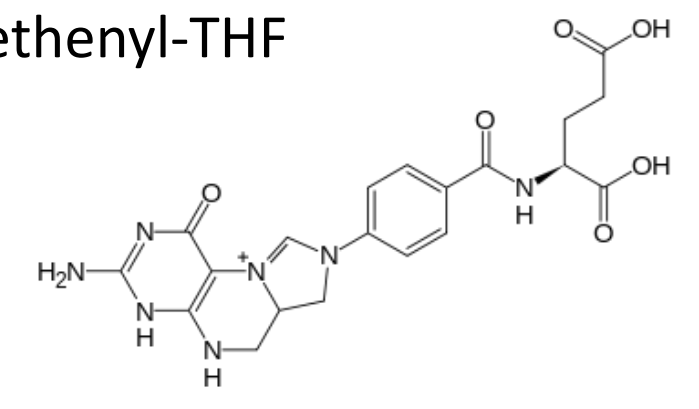

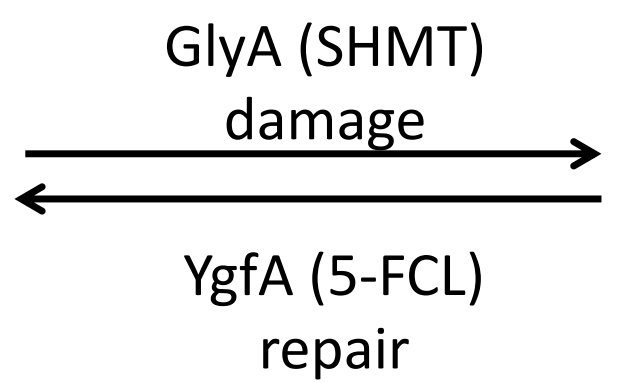

$$
\text { -Formyltetrahydrofolate }
$$

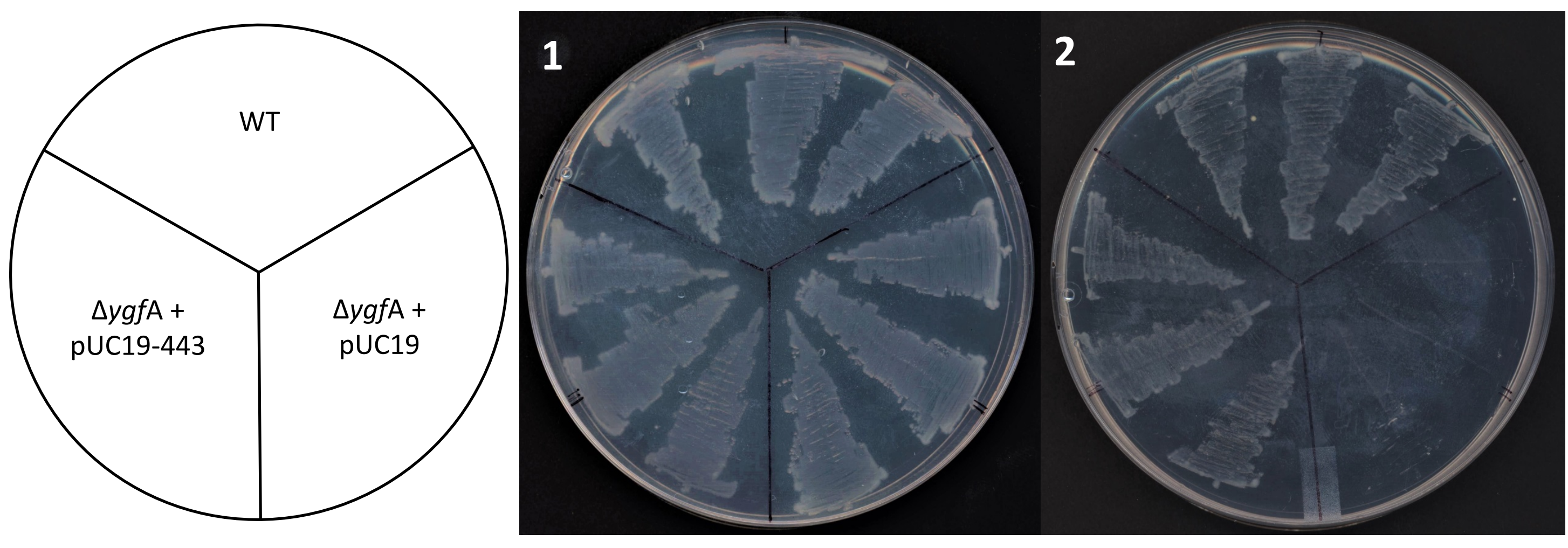




\section{Figure 2}

A

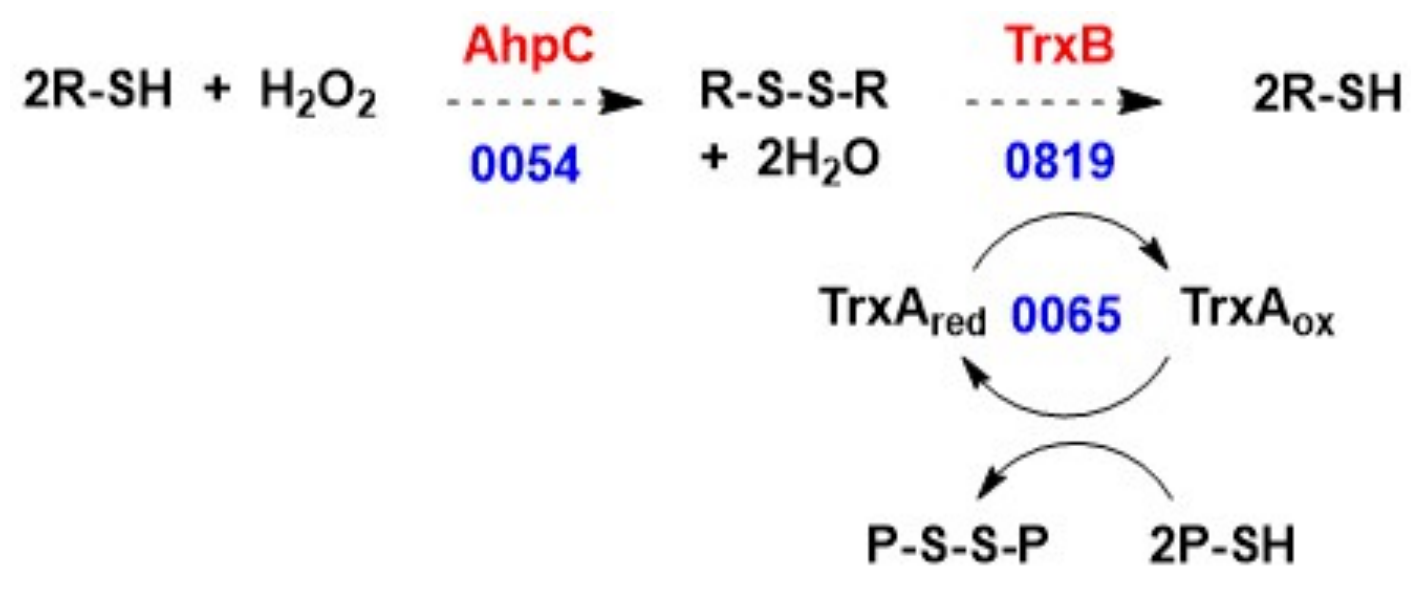

B

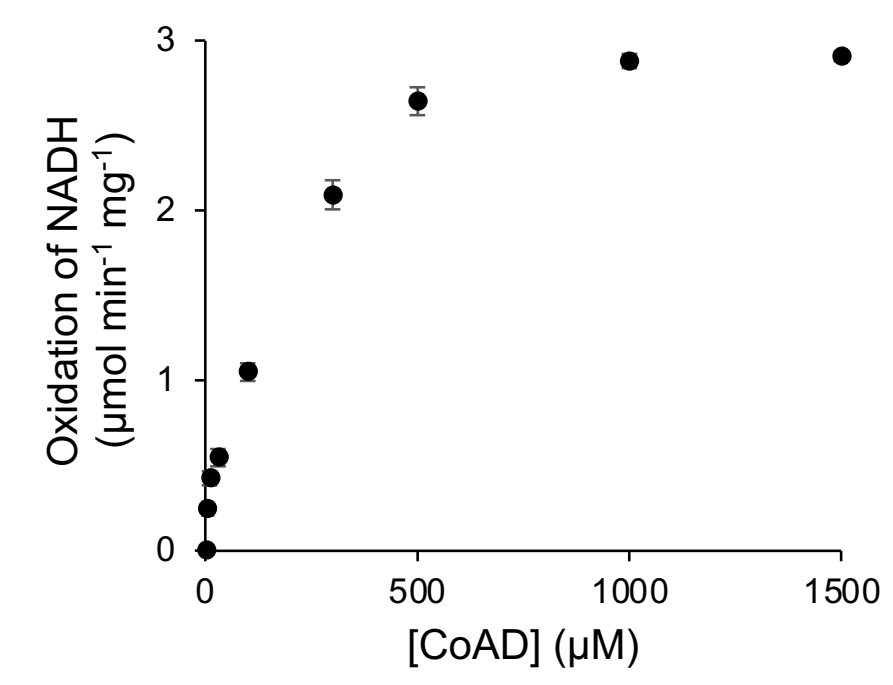

C

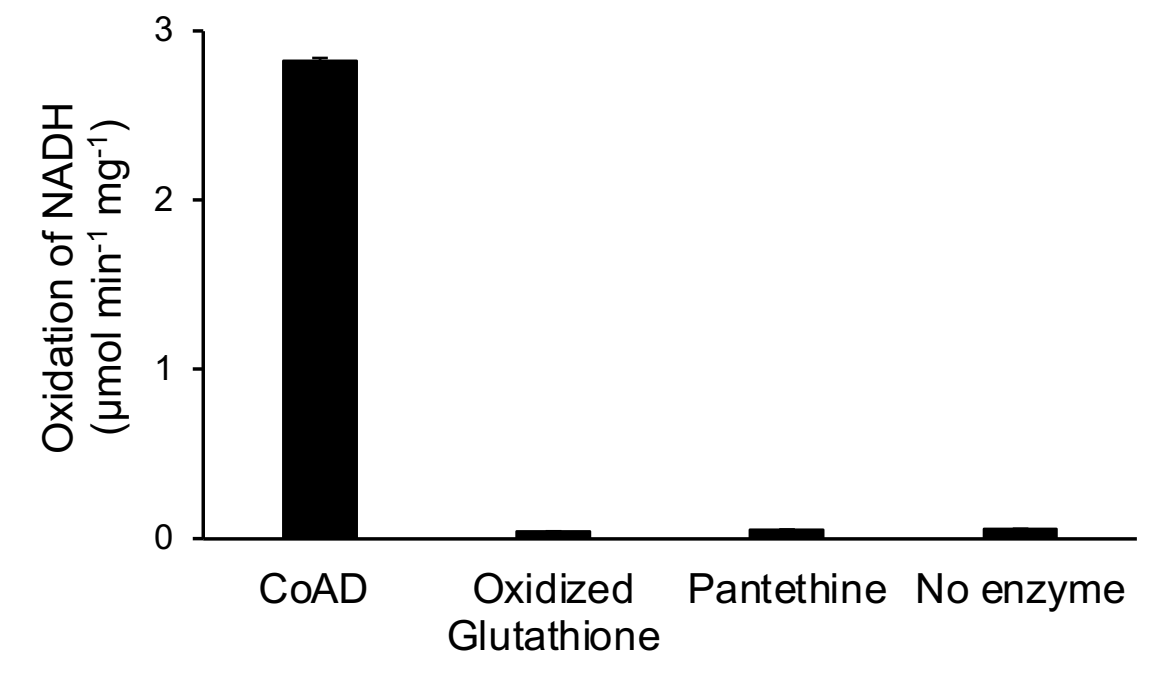

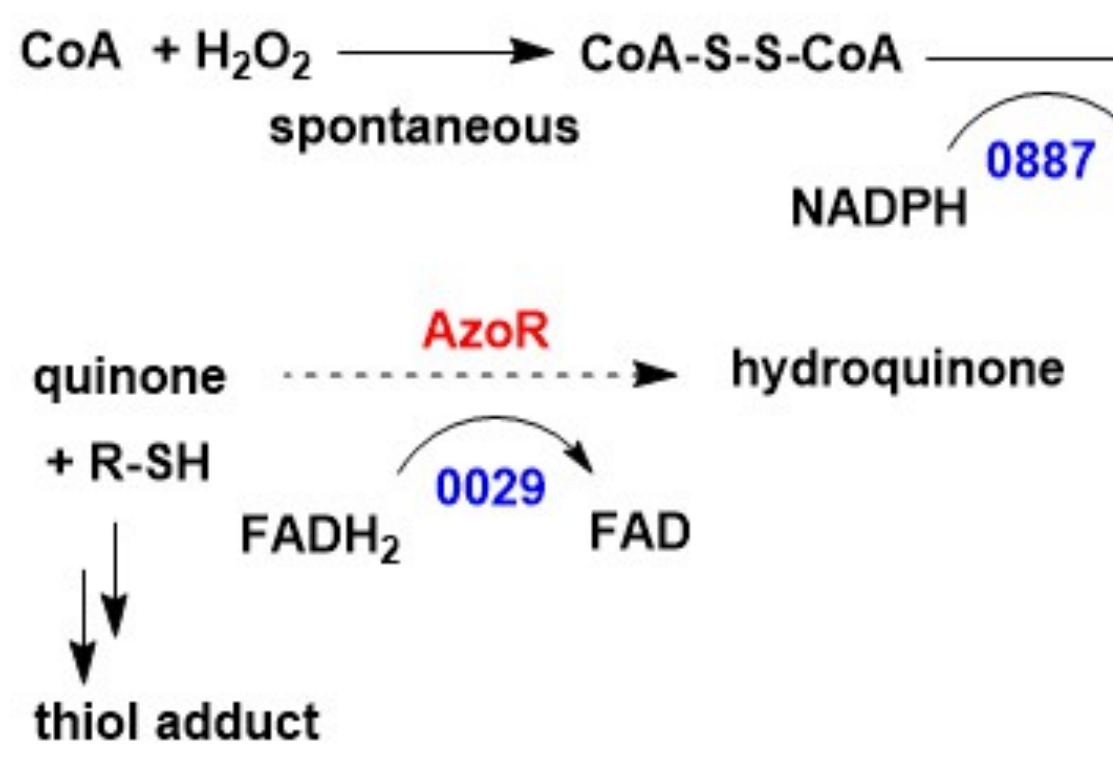




\section{Figure 3}

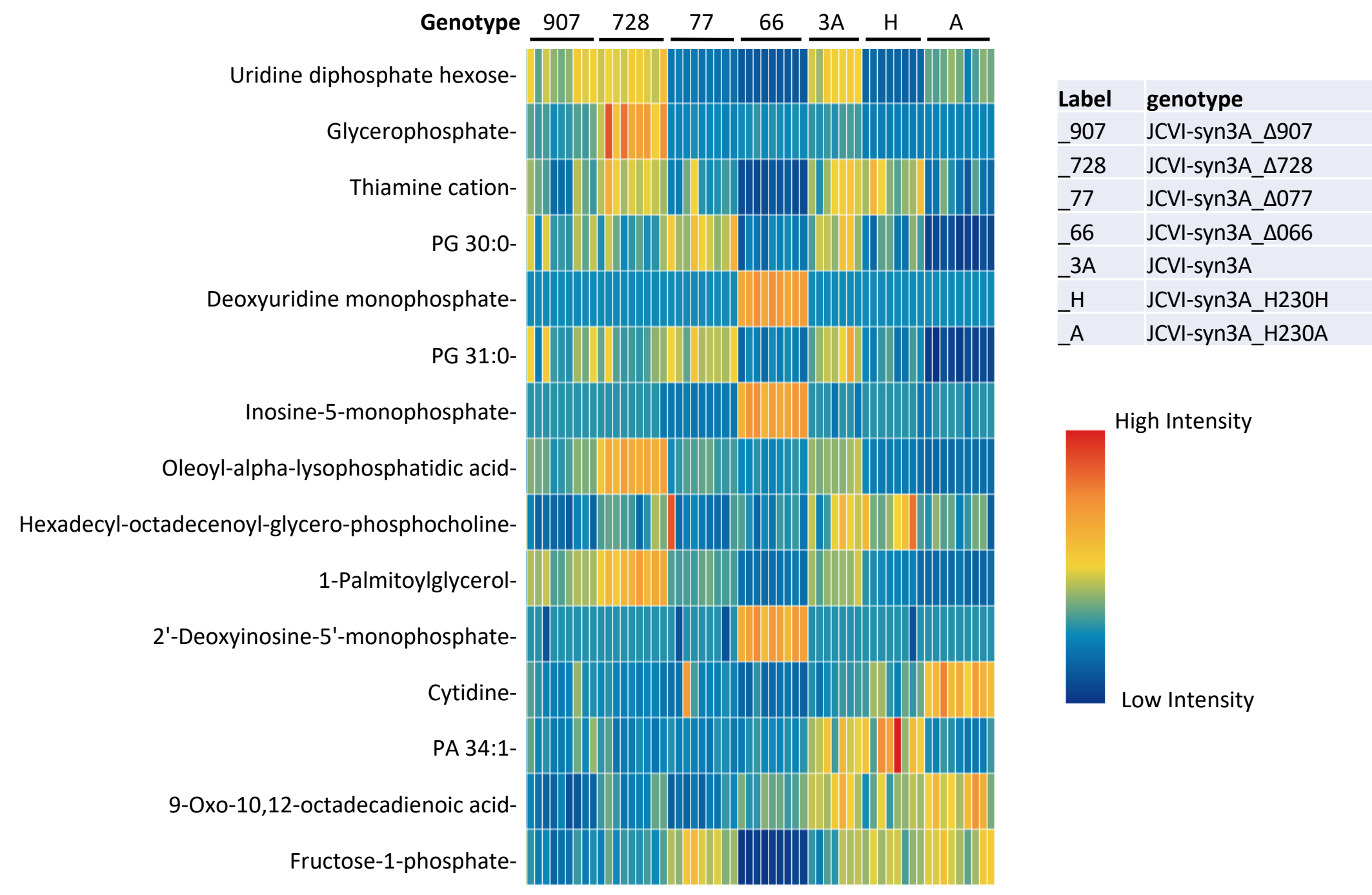




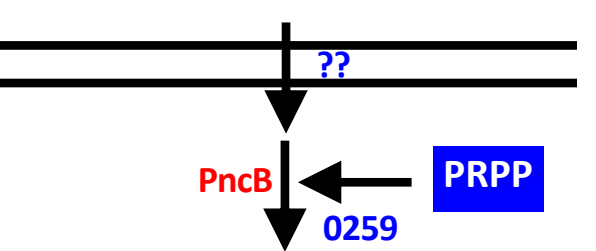

Nicotinate-ribonucleotide (NaMN)

Nicotinate-adenine dinucleotide (NaAD)

$$
\text { Nade } \underset{0378}{\leftarrow} \mathrm{Gln}
$$

B

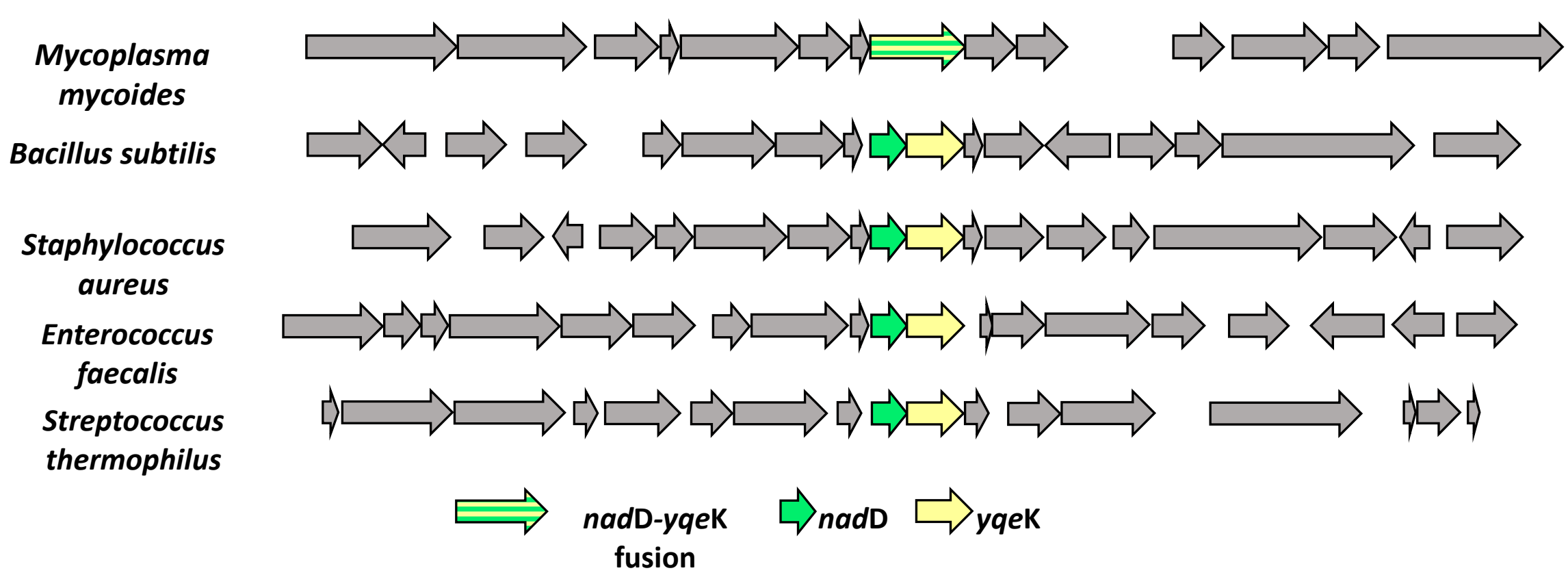


A

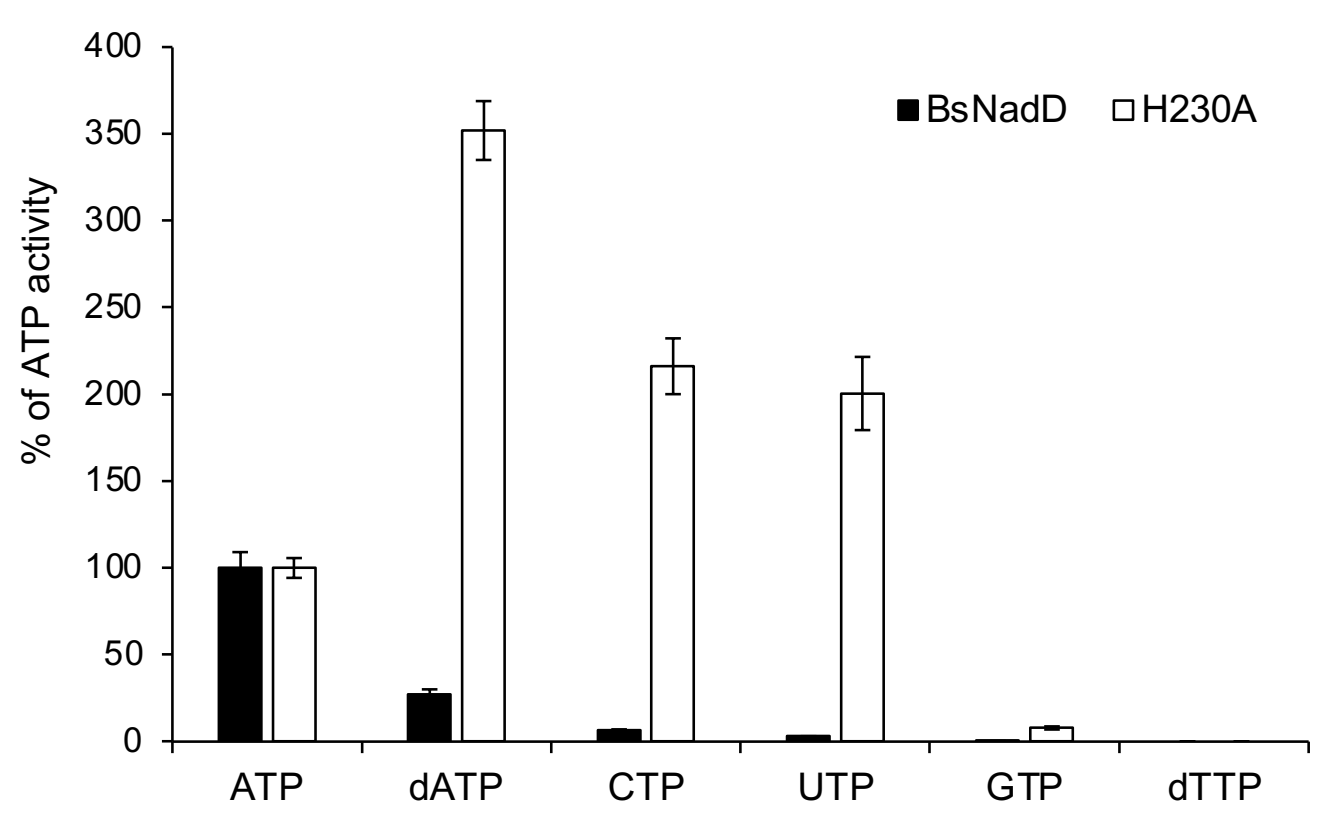

B

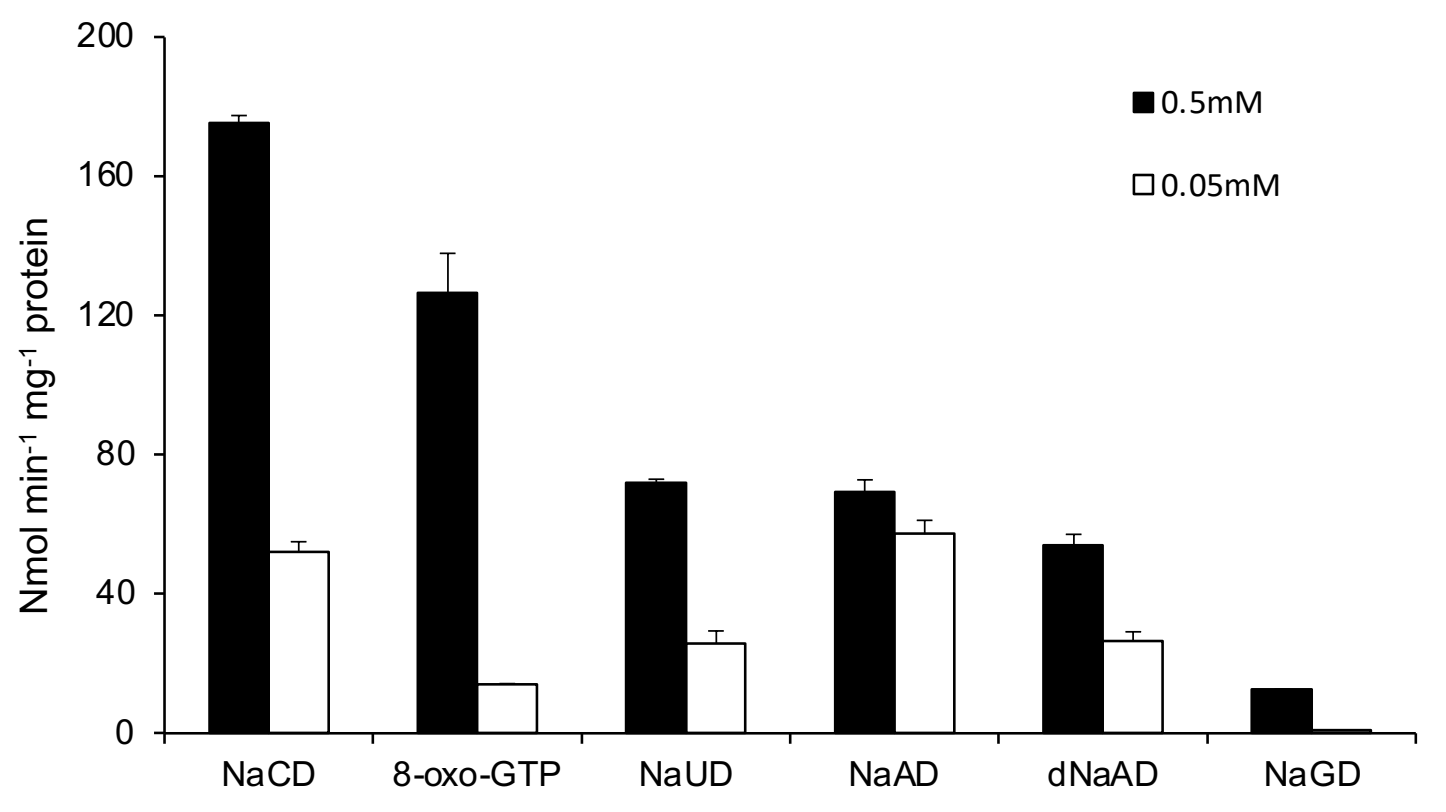

C

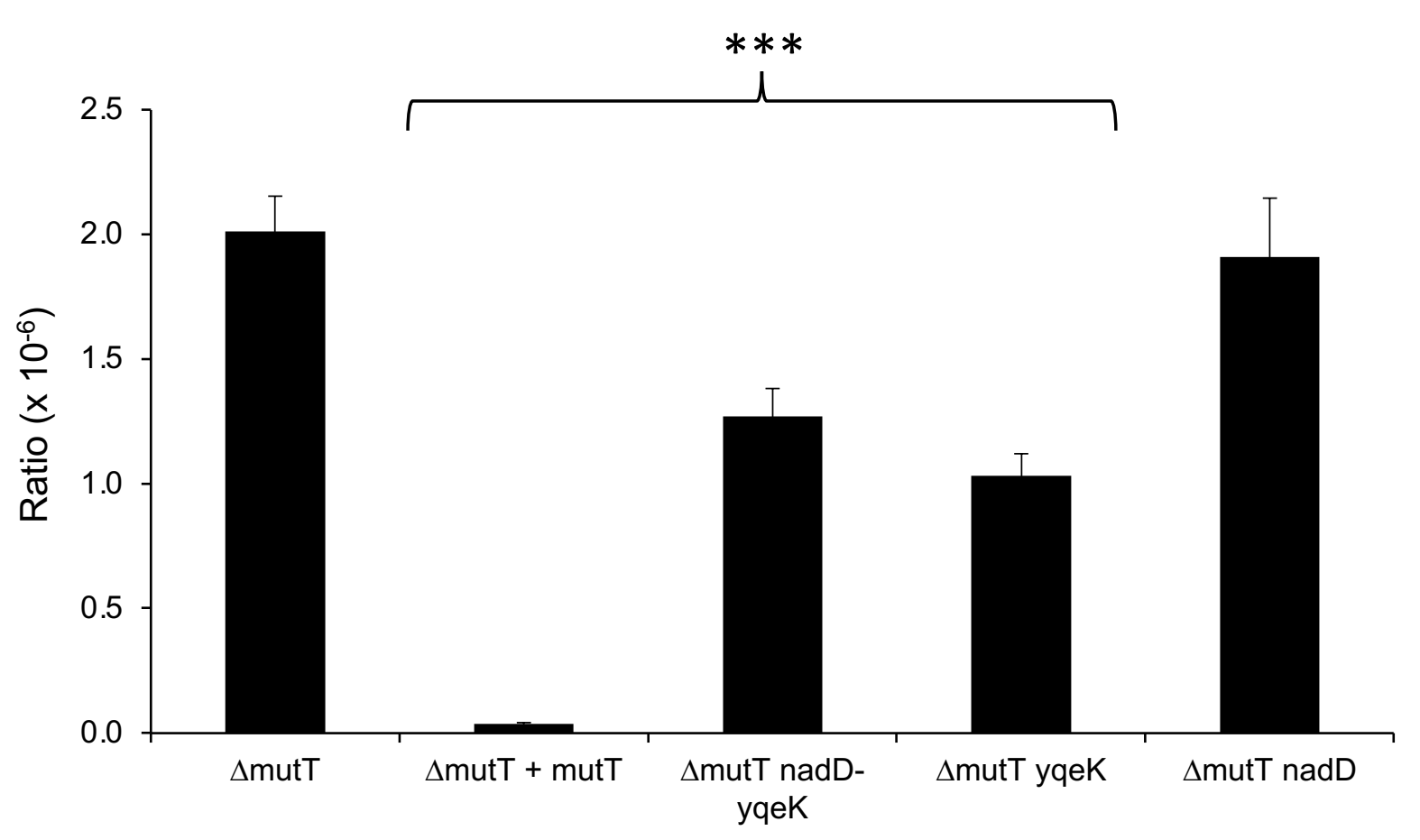

Figure 5 


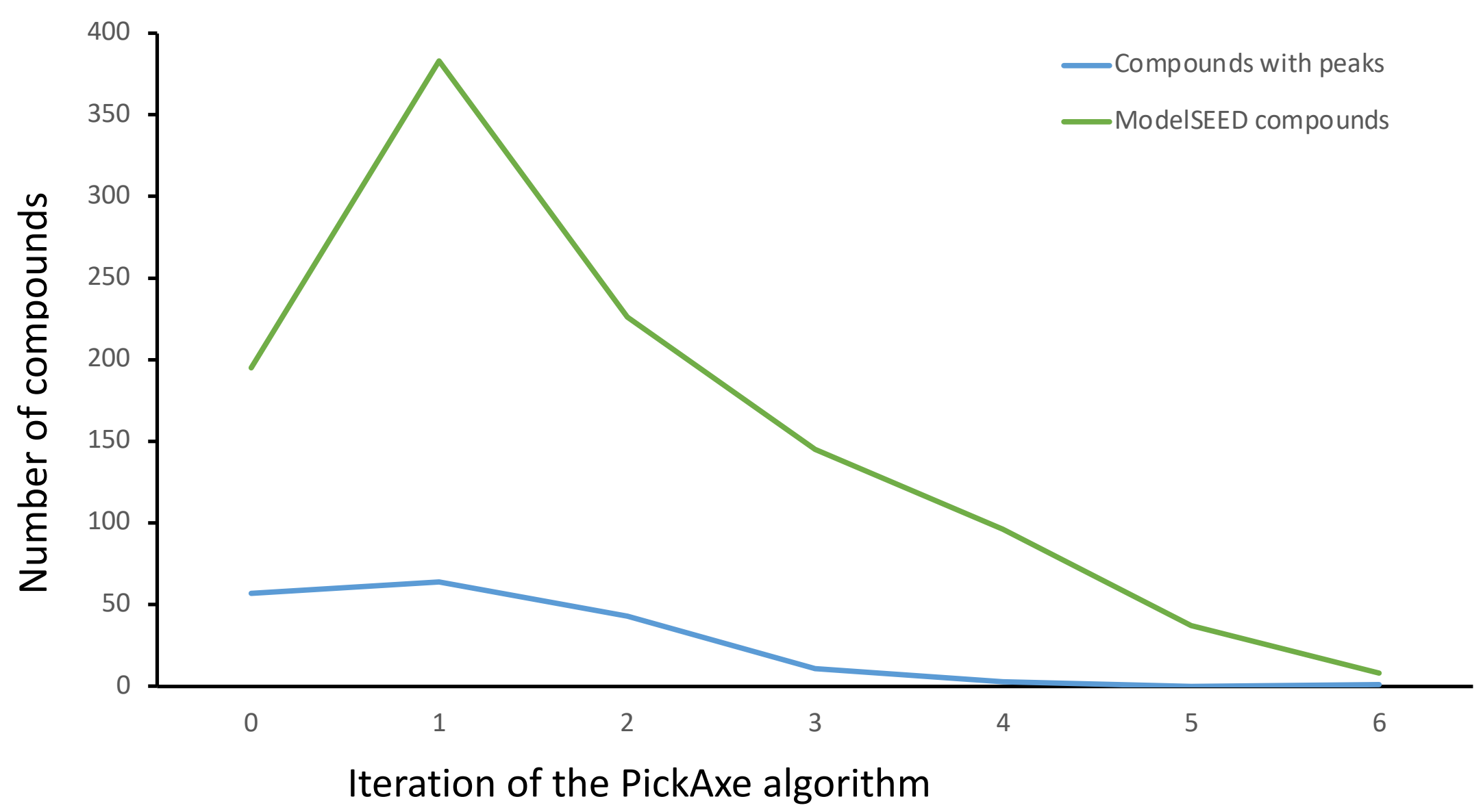




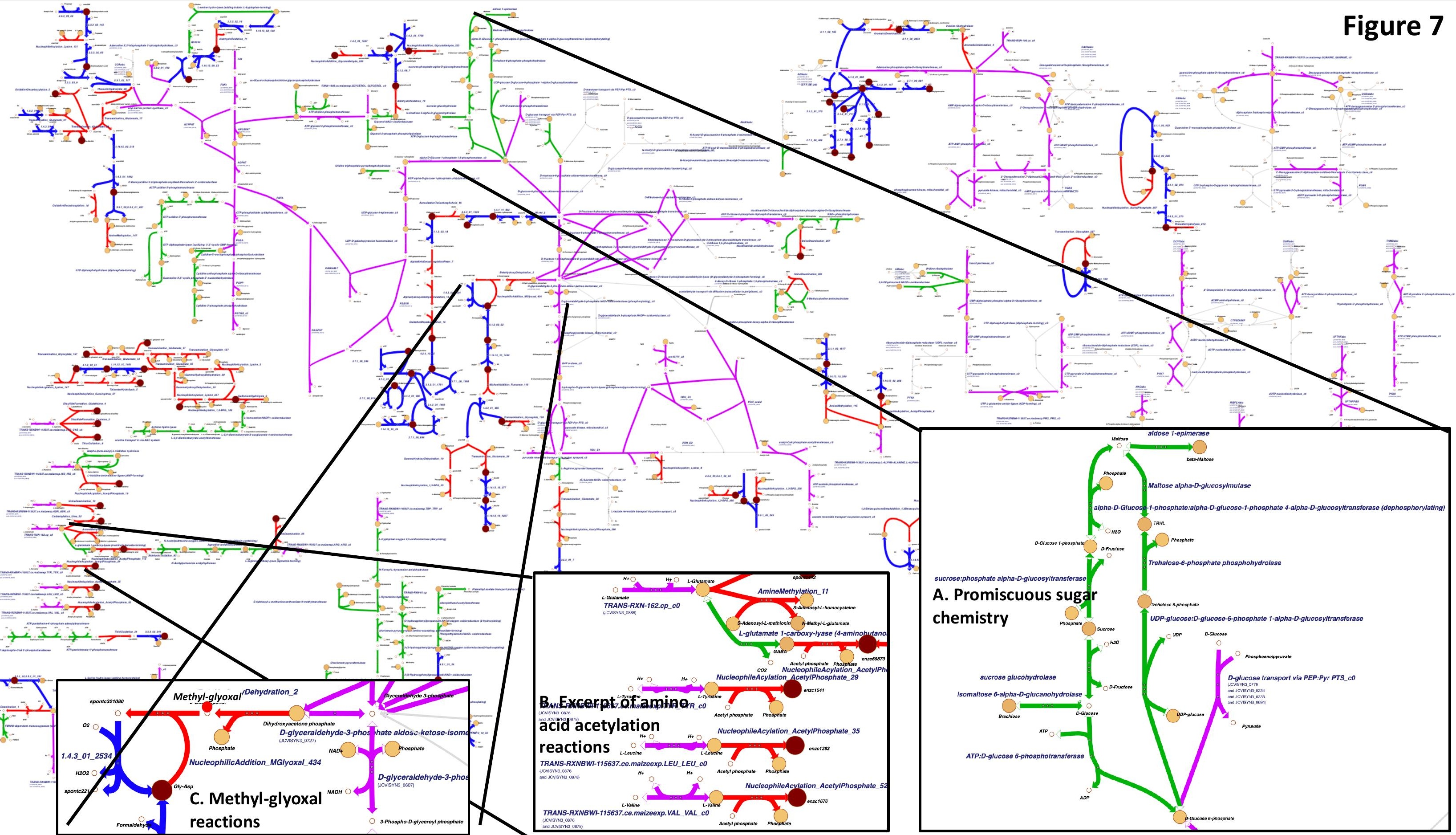




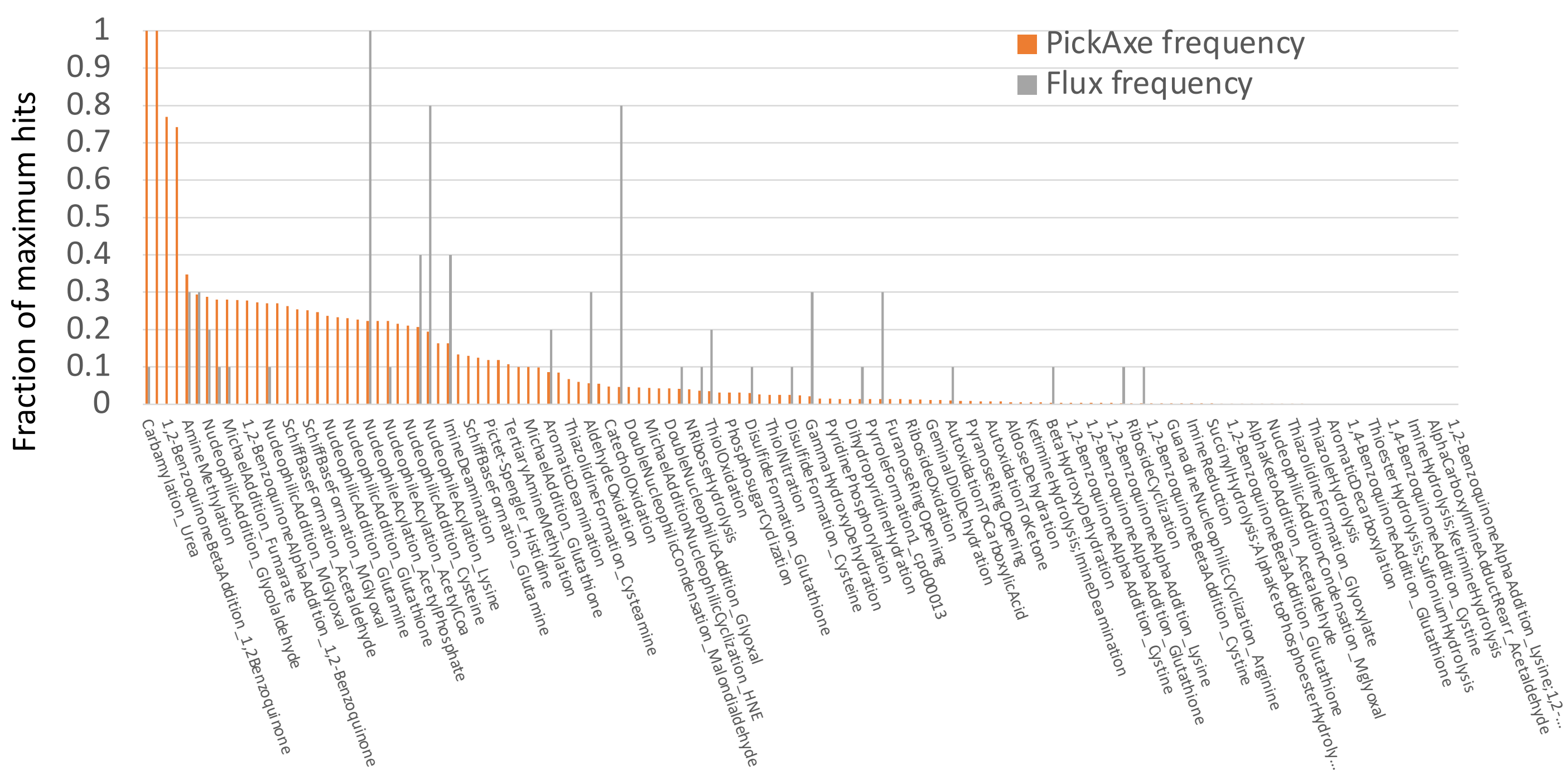

Reaction rule 
A

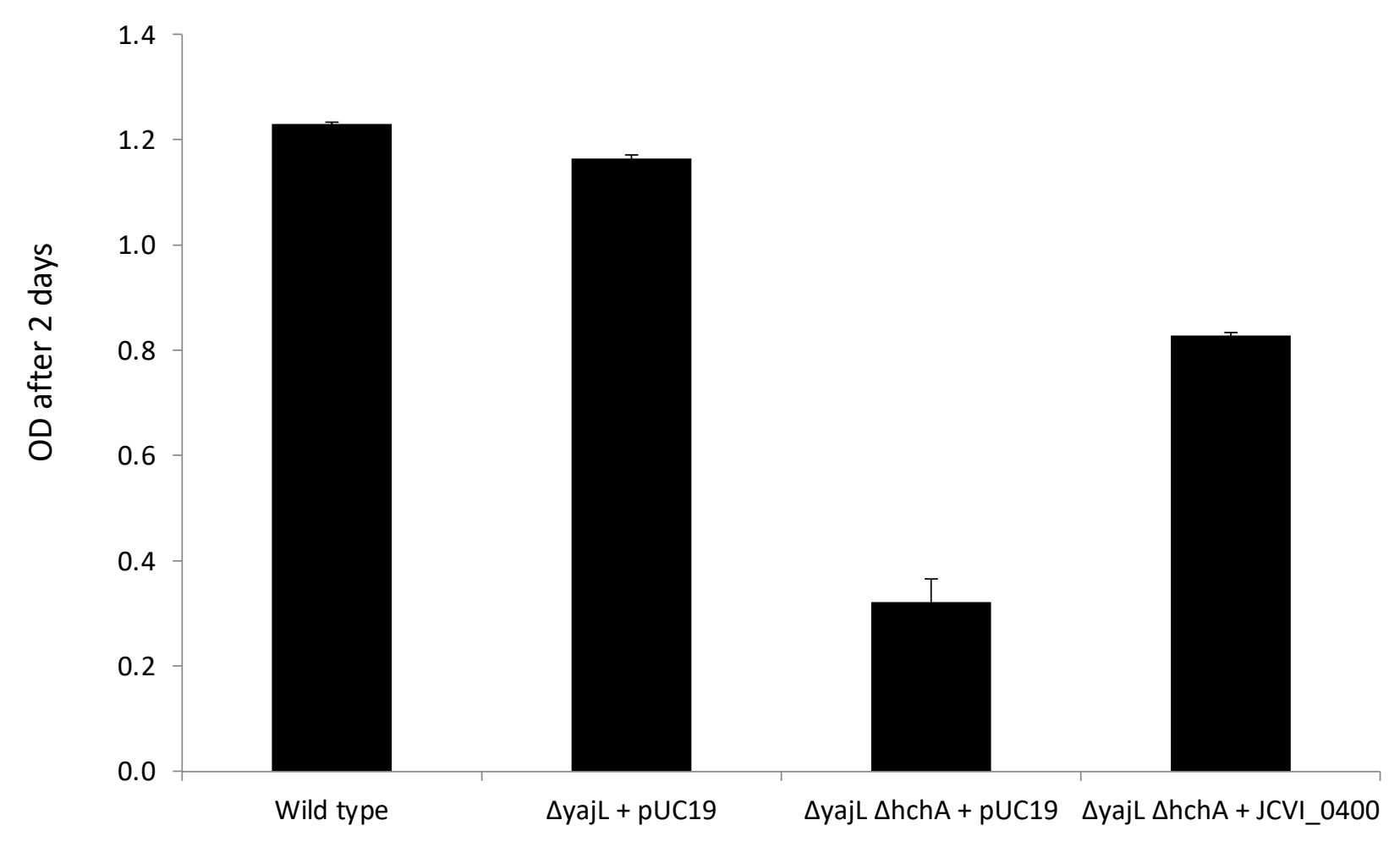

B

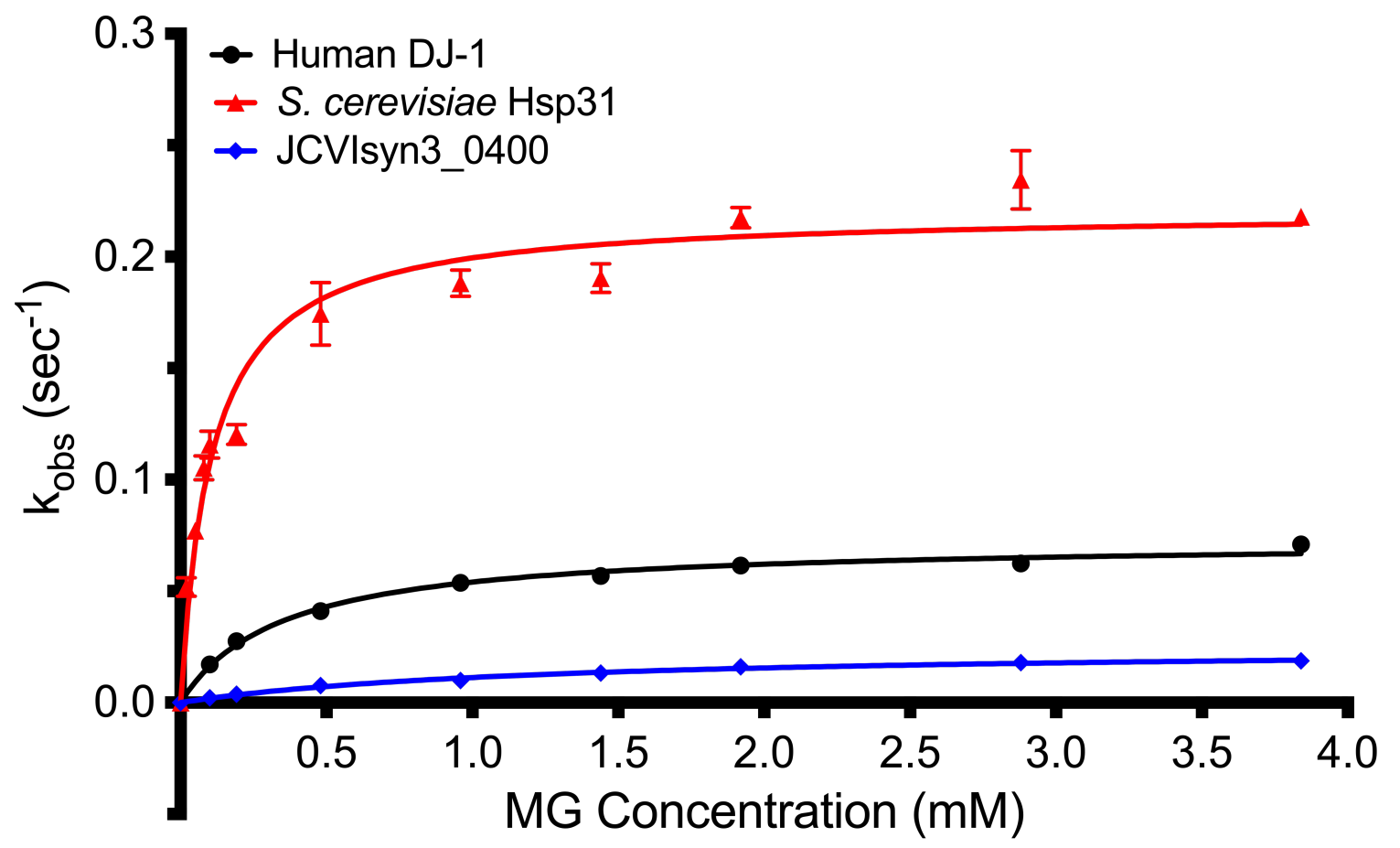

